\section{Mitochondria form contact sites with the nucleus to couple prosurvival retrograde response}

\author{
Radha Desai ${ }^{1{ }^{\dagger}}{ }^{\dagger}$, Daniel A. East ${ }^{1 *}$, Liana Hardy ${ }^{1}{ }^{\ddagger}$, Danilo Faccenda ${ }^{1}$, Manuel Rigon ${ }^{1}$, \\ James Crosby ${ }^{1}$, María Soledad Alvarez ${ }^{1 \S}$, Aarti Singh ${ }^{1}$, Marta Mainenti ${ }^{1} \|$, Laura Kuhlman Hussey ${ }^{1}$, \\ Robert Bentham ${ }^{2}$, Gyorgy Szabadkai ${ }^{2,3,4}$, Valentina Zappulli ${ }^{5}$, Gurtej K. Dhoot ${ }^{1}$, Lisa E. Romano ${ }^{6}$, \\ Dong Xia ${ }^{1}$, Isabelle Coppens ${ }^{7}$, Anne Hamacher-Brady ${ }^{7}$, J. Paul Chapple ${ }^{6}$, Rosella Abeti ${ }^{8}$, \\ Roland A. Fleck ${ }^{9}$, Gema Vizcay-Barrena ${ }^{9}$, Kenneth Smith ${ }^{10}$, Michelangelo Campanella ${ }^{1,2}$
}

\begin{abstract}
Mitochondria drive cellular adaptation to stress by retro-communicating with the nucleus. This process is known as mitochondrial retrograde response (MRR) and is induced by mitochondrial dysfunction. MRR results in the nuclear stabilization of prosurvival transcription factors such as the nuclear factor kappa-light-chain-enhancer of activated $B$ cells (NF-KB). Here, we demonstrate that MRR is facilitated by contact sites between mitochondria and the nucleus. The translocator protein (TSPO) by preventing the mitophagy-mediated segregation o mitochonria is required for this interaction. The complex formed by TSPO with the protein kinase $A$ (PKA), via the A-kinase anchoring protein acyl-CoA binding domain containing 3 (ACBD3), established the tethering. The latter allows for cholesterol redistribution of cholesterol in the nucleus to sustain the prosurvival response by blocking NF-kB deacetylation. This work proposes a previously unidentified paradigm in MRR: the formation of contact sites between mitochondria and nucleus to aid communication.
\end{abstract}

\section{INTRODUCTION}

Mitochondria actively participate in the reprogramming of mammalian cells $(1,2)$. In response to endogenous or exogenous perturbations, they retro-communicate with the nucleus to drive the transcription of genes. This pathway of signaling is known as the mitochondrial retrograde response (MRR) and is exploited in the pathogenesis of uncontrolled cellular proliferation (3). In cancer, prosurvival transcription factors (TFs) like the nuclear factor kappa-light-chain-enhancer of activated B cells (NF- $\mathrm{KB})(4-7)$ are stabilized by the hyperproduction of reactive oxygen species (ROS) generated from dysfunctional mitochondria. Impaired cell signaling and nuclear accumulation of $\mathrm{NF}-\kappa \mathrm{B}$ are the acknowledged readouts of active MRR in mammals

\footnotetext{
'Department of Comparative Biomedical Sciences, The Royal Veterinary College, University of London, Royal College Street, London NW1 OTU, UK. ${ }^{2}$ Department of Cell and Developmental Biology, Consortium for Mitochondrial Research (CFMR), University College London, Gower Street, London WC1E 6BT, UK. ${ }^{3}$ Department of Biomedical Science, University of Padua, Via Ugo Bassi, 35131 Padua, Italy. ${ }^{4}$ Francis Crick Institute, Midland Road, London NW1 AT, UK. ${ }^{5}$ Department of Comparative Biomedicine and Food Sciences, University of Padua, Viale dell'Universita' 16, 35020 Legnaro (PD), Italy. ${ }^{6}$ William Harvey Research Institute, Barts and the London School of Medicine, Queen Mary University of London, London EC1M 6BQ, UK. ${ }^{7}$ W. Harry Feinstone Department of Molecular Microbiology and Immunology, Johns Hopkins University Baltimore, Baltimore, MD 21205, USA. ${ }^{8}$ Ataxia Centre, Department of Clinical and Movement Neurosciences, UCL Queen Square Institute of Neurology, Queen Square, London WC1N 3BG, UK. ${ }^{9}$ Centre for Ultrastructural Imaging, King's College London, London SE1 1UL, UK. ${ }^{10}$ Pathobiology and Population Sciences, The Royal Veterinary College, Hawkshead Lane, North Mymms, Hatfield, Hertfordshire AL9 7TA, UK.

*These authors contributed equally to this work.

tPresent address: Discovery Research MRL UK, MSD, The London Bioscience Innovation Centre (LBIC), 2 Royal College Street, London NW1 ONH, UK.

¥Present address: Department of Biochemistry, University of Cambridge, Cambridge CB2 1QW, UK.

§Present address: Vascular Biology Lab, School of Medicine-UNCuyo, Institute of Medicine and Experimental Biology of Cuyo IMBECU-CONICET CCT, 5500 Mendoza, Argentina.

||Present address: Utah Veterinary Diagnostic Laboratory (UVDL), Utah State University, 950 E 1400 N, Logan, UT 84341, USA.

ПCorresponding author. Email: mcampanella@rvc.ac.uk
}

$(8,9)$. In these species, the lack of homology with the yeast Rtg1/Rtg2/ Rtg3 system (10) entails the engagement of a more complex framework of regulators and executors (11) when mitochondria communicate with the nucleus. In keeping with this, the recent identification of MRR molecules $(8,9)$ failed to explain the interplay between mitochondria and nucleus during stress. Aspects intimately involved in the organelle physiopathology such as (i) dynamic remodeling (ii) and trafficking of lipids had been unexplored, and for these, we postulated a catalytic role in the MRR signaling. Abnormalities in lipid metabolism are distinctive features of cancer cells, which vastly rely on MRR (12), and in these, the increased mitochondrial network size following the stabilization of profusion proteins (e.g., OPA-1, Mnf1, 2, ATPIF1) (13-15) mediates cytoprotective resistance to organelle disassembly. Enlarged mitochondria by occupying the surrounding cytosolic space would therefore hold a greater chance of establishing proximity events with other organelles to generate hotspots of communication. It is well established that hyperfused, remodeled mitochondria interact with the endoplasmic reticulum (ER), while unexplored is whether they could become physically coupled with the nucleus to expedite the execution of MRR.

To test the hypothesis of domains of communication between mitochondria and nucleus during MRR, we traced the molecular physiology of the outer mitochondrial membrane (OMM)-based protein TSPO (18-kDa translocator protein) (16). This stress-responsive protein is up-regulated in many cancers (17-19) and linked to antiapoptotic activity. Its pharmacological ligands allow its selective targeting to coadjuvate chemotherapy and in vivo tracing of metastatic cells $(20,21)$. Once accumulated on the OMM, TSPO represses mitophagy (22) and deregulates mitochondrial buffering capacity for $\mathrm{Ca}^{2+}$ and production of adenosine $5^{\prime}$-triphosphate (ATP) (23) originated from the oxidative phosphorylation. TSPO recruits and docks on mitochondria the A-kinase anchoring protein acyl-coenzyme A (CoA) binding domain containing 3 (ACBD3) and protein kinase $\mathrm{A}$ (PKA) $(23,24)$, which complexes with the nucleus via the A-kinaseanchoring protein AKAP95 (A-kinase-anchoring protein 95$)(25,26)$. 
Breast cancer, in which TSPO is overexpressed $(27,28)$, has modified signaling (29) and metabolism (30) of cholesterol, which is trafficked by TSPO to fuel the synthesis of pregnenolone $(29,31)$ within the mitochondrial matrix (32). Cholesterol, its intermediates, and their enzymatic catalysts such as the aromatase enzyme CYP19A1 are involved in the oncogenic reprogramming $(33,34)$. All of which make TSPO an ideal means to insight the interplay between organelle morphology and lipid metabolism in the MRR.

Using cellular and ultrastructural means of analysis combined with advanced protocols of imaging, we show that TSPO is required to establish contact sites between mitochondria and nucleus during MRR, allowing for redistribution of cholesterol that deacetylates $\mathrm{NF}-\kappa \mathrm{B}$, completing the prosurvival response. A novel regulatory axis in the MRR that operates via the (i) formation of contact sites (ii) and redistribution of cholesterol between mitochondria and nucleus is therefore outlined. This evidence advances our understanding of a key process in mammalian pathophysiology and details a physical and metabolic coupling between mitochondria and nucleus that is previously unknown.

\section{RESULTS \\ TSPO facilitates the stabilization of NF-кB into the nucleus during MRR}

The first step of our analysis consisted in assessing the correlation between the candidate protein TSPO and the nuclear translocation of the prosurvival transcriptional factor NF- $\kappa \mathrm{B}(35,36)$, which is an acknowledged driving pathway of the MRR. We inspected primary human samples of mammary gland tumors, reporting a positive association between TSPO level, aggressiveness of lesions (Fig. 1, A and $\mathrm{B}$ ), and $\mathrm{NF}-\kappa \mathrm{B}$ level into the nucleus (Fig. $1, \mathrm{C}$ and $\mathrm{D}$ ). In line with this, transcriptomic analysis of $>600$ samples of patients with breast cancer in The Cancer Genome Atlas (TCGA) showed that higher levels of active NF- $\kappa \mathrm{B}$ are correlated with increased TSPO expression in the more aggressive forms of breast cancer (HER positive and basal types) (Fig. 1, E and F). This is independent of mutations in TSPO, whose increased expression is an underlying feature in the neoplasia of the mammary gland retained across species (fig. S1, A to E).

To elucidate the nature of this oncogenic interplay between TSPO and NF- $\kappa \mathrm{B}$, we moved the analysis in vitro and compared two epithelial human breast cancer cell lines, MCF-7, which features low TSPO levels, and the more aggressive MDA-MB-231 (henceforth referred to as MDA), which instead expresses high TSPO (29) levels (Fig. 1, G and H). To engage the MRR, we used the mitochondrial stressor staurosporine (STS), which induces the remodeling of mitochondria around the nucleus (as shown in MDA; Fig. 1, J and K) and, by promoting TSPO expression (fig. S1, F and G), facilitates the nuclear accumulation of NF- $\kappa \mathrm{B}$ (Fig. $1, \mathrm{~L}$ to N). The involvement of TSPO in this pathway was confirmed by the failure of STS to induce $\mathrm{NF}-\kappa \mathrm{B}$ retro-translocation in MDA cells transiently knocked down for TSPO (-TSPO). The outcome of the increased NF- $\kappa \mathrm{B}$ retrotranslocation was corroborated by analyses of the transcriptional profile of the NF- $\mathrm{KB}$-regulated prosurvival genes c-FLIP and Bcl-2 (Fig. 1, Q to S). Immunocytochemical analysis further highlighted the degree of nuclear residency of NF- $\kappa$ B upon STS treatment and how pruning of the mitochondrial network via pharmacological activation of mitophagy by the p62/SQSTM1-mediated mitophagy inducer $(\mathrm{PMI})(37,38)$ reduces the degree of $\mathrm{NF}-\kappa \mathrm{B}$ translocation (Fig. 1, $\mathrm{O}$ and $\mathrm{P}$ ). By reducing the size of the mitochondrial network,
PMI limits the cytosolic space occupancy by mitochondria (fig. S1J) and sensitizes human, feline, and canine breast cancer cells to STS-induced apoptosis (fig. S1, K to M). The integrity of the mitochondrial network, which is reflected by TSPO overexpression and accumulation on the OMM, is therefore pivotal to stabilize the retrotranslocation and transcriptomic capacity of NF- $\kappa \mathrm{B}$ during MRR activation.

\section{Targeting TSPO and its cholesterol binding capacity dictates programmed cell death}

Like PMI, treatment of MDA cells with the TSPO ligand PK11195 (Table 1), which competes with the cholesterol binding domains of the protein (39), inhibits NF- $\kappa \mathrm{B}$ retro-translocation (Fig. 2, A and B). Equally efficient in this is the cholesterol synthesis inhibitor pravastatin (PVS) (Fig. 2C). This implies the existence of a regulatory hierarchy between TSPO, cholesterol, and NF- $\kappa \mathrm{B}$ in regulating MRRmediated evasion of cell death, which we further assessed by enrolling MDA cells stably knocked out for NF- $\kappa \mathrm{B}$ (NF- $\mathrm{KB} \mathrm{KO})(40)$. In these cells, an additive affect was given by the repression of TSPO concomitant with that of NF- $\mathrm{KB}$ during STS treatment (Fig. 2D). Knocking down TSPO (TSPO KD) in MDA cells competent for NF- $\kappa \mathrm{B}$ was thus able to (i) increase sensitivity to apoptosis (Fig. 2E) [an effect retained across species (fig. S1, H and I)], (ii) reduce cellular proliferative capacity (Fig. 2F), and (iii) facilitate the accumulation of the proapoptotic protein Bax on mitochondria (Fig. 2, G and H): Last, (iv) it also increased the release of cytochrome $\mathrm{C}$ (Cyt c) in the cytosol (Fig. 2, I and J). An array of effects acknowledges a role for TSPO wider than its interplay with NF- $\mathrm{KB}$. In keeping with this, TSPO ligands (Table 1) tangibly promoted susceptibility to chemically induced cell death, thereby exacerbating not only STS effects (Fig. 2K) but also that of doxorubicin and vincristine (Fig. 2, L and M) in both two-dimensional (2D) and 3D cultures (fig. S3A).

\section{Endocrine therapy resistance up-regulates TSPO, which defines the juxtaposition of mitochondria with the nucleus}

MCF-7 cells, which are constitutively low in TSPO expression, gained resistance to STS-induced apoptosis when wild-type TSPO (WT TSPO) was expressed (Fig. 3B). Although, the mutant clone of the protein, devoid of the CRAC cholesterol binding domain (TSPO $\triangle$ CRAC), failed to protect from the treatment (Fig. 3, A and B). This evidence further argued that the cholesterol-binding capacity of TSPO is crucial to drive resistance against chemically induced cell death. In keeping with this, TSPO level of expression increases in MCF-7 cells that have developed resistance to tamoxifen (MCF7T) (6), or in which cholesterol biosynthesis sparked following long-term estrogen deprivation (MCF7-LTED) (Fig. 3, D and E) $(6,34,35)$.

Treatment with the TSPO ligand FGIN-127 (hereafter referred to as FGIN) impairs handling of cholesterol by the protein-like PK11195 (Table 1), thereby operating as potential chemical deterrent for the nuclear accumulation of the estrogen receptor $\alpha(\mathrm{ER} \alpha)$. The nuclear internalization of the latter is key for the transition of epithelial breast tumor toward a more aggressive phenotype, leading to therapeutic failure of tamoxifen $(41,42)$ treatment. FGIN increases susceptibility to STS-induced cell death (Fig. 3F) as well as reduces $\mathrm{ER} \alpha$ association with the nucleus (fig. S3, D and E). In line with these observations, big-data analysis revealed that TSPO is overexpressed in cohorts of relapsed patients with breast cancer, in which endocrine therapy (ET) was no longer effective and cholesterol metabolism escalated (fig. S3, B and C). 


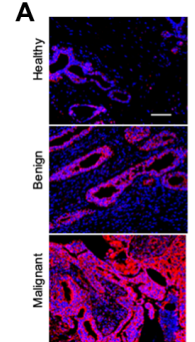

E

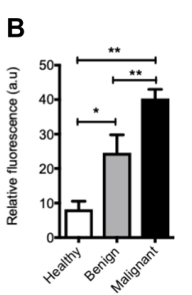

F

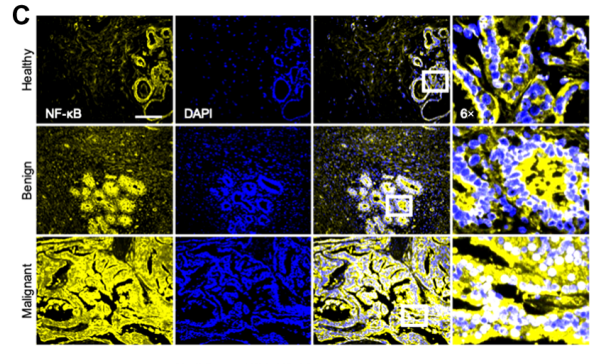

G MCF-7 MDA-MB-231
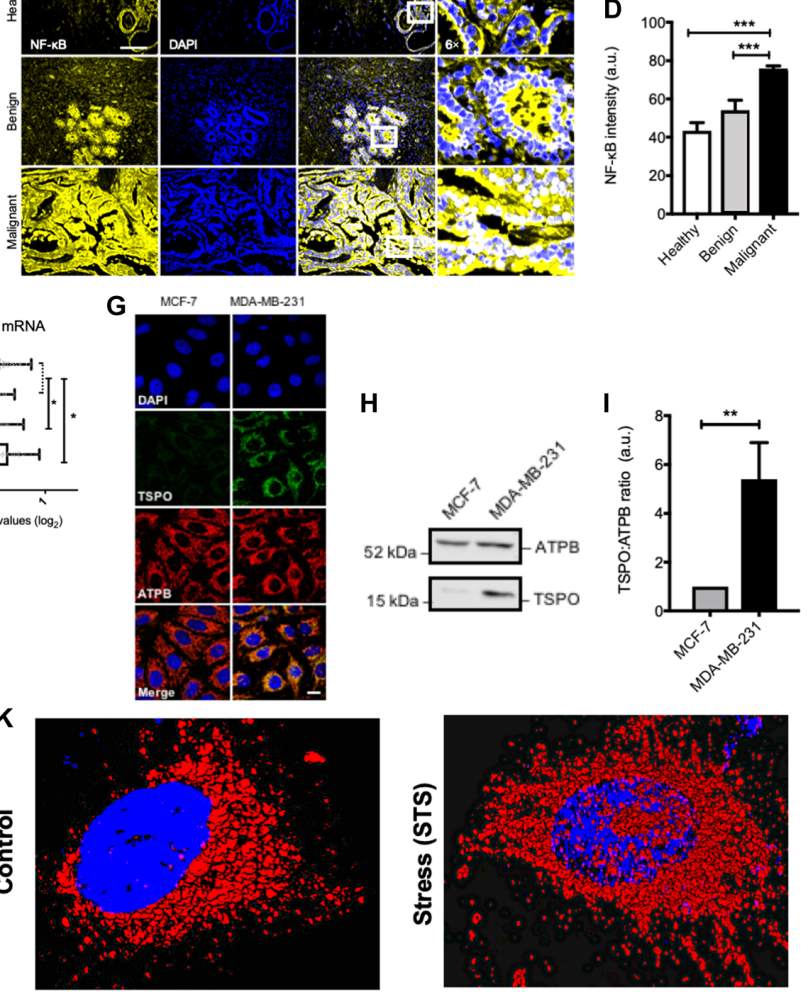

$\mathbf{N}$

M

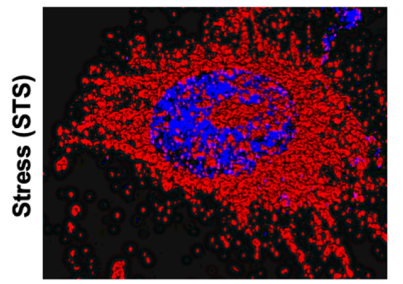

H

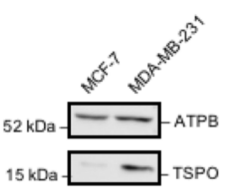

$\mathbf{L}$
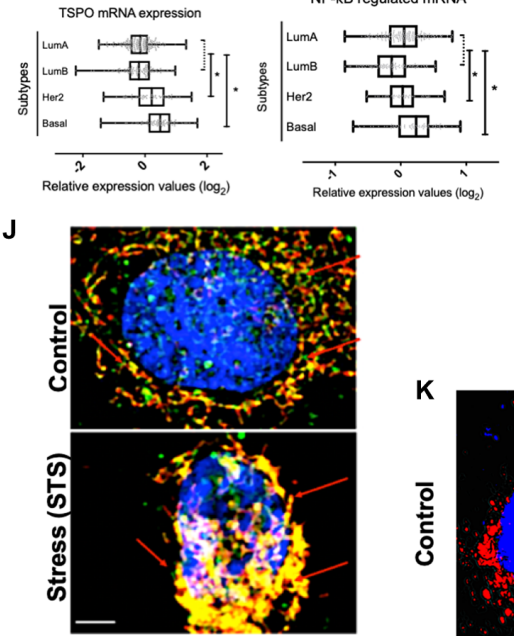

Nucleus: blue; VDAC: green; TSPO: red
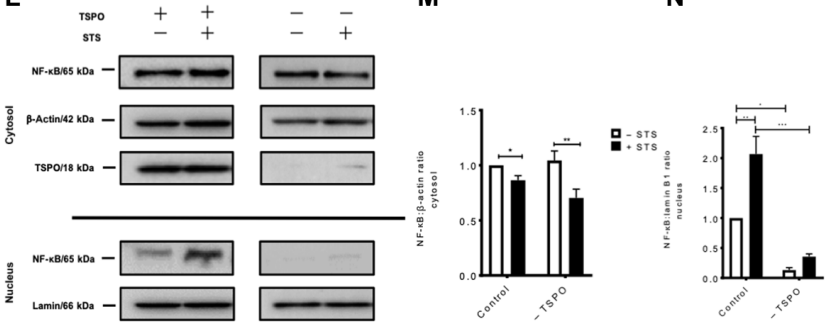

$\mathbf{P}$

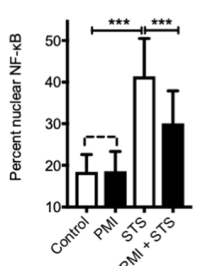

Q

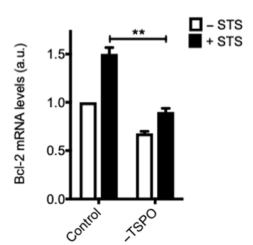

R

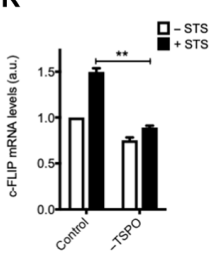

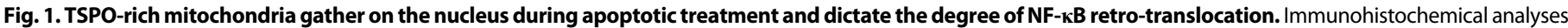

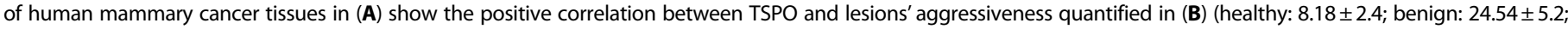

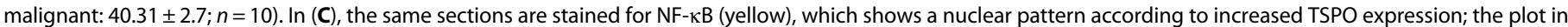

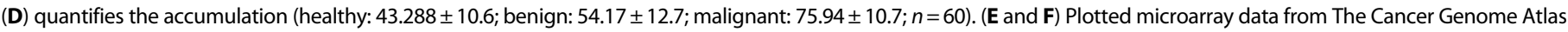

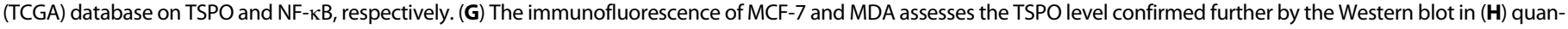

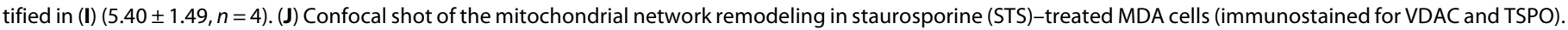

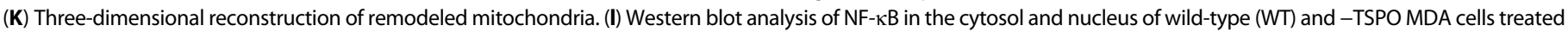

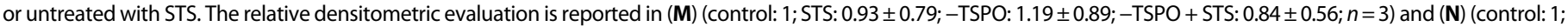

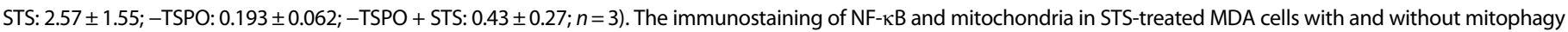

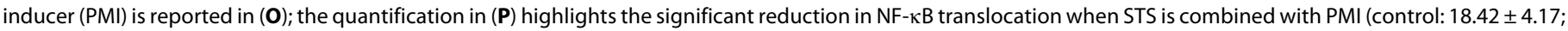

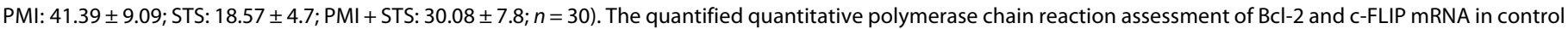

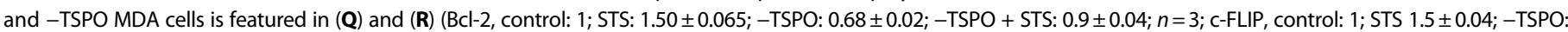

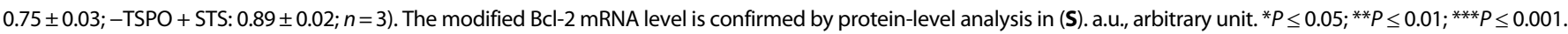


Table 1. List of the chemical structures of the compounds used in the study.

Compound

IUPAC (International Union of Pure and Applied Chemistry)

Structure

PK11195

1-(2-Chlorophenyl)- $N$-methyl- $N$-(1-methylpropyl)-3-isoquinolinecarboxamide

FGIN (FGIN-1-27)

$\mathrm{N}, \mathrm{N}$-dihexyl-2-(4-fluorophenyl)indole-3-acetamide

Ro5 (Ro5-4864)

7-Chloro-5-(4-chlorophenyl)-1-methyl-3H-1,4-benzodiazepin-2-one

\section{Staurosporine}

Doxorubicin

Rotenone

PMI

Vincristine

Tamoxifen
(2S,3R,4R,6R)-3-methoxy-2-methyl-4-(methylamino)-29-oxa-1,7,17triazaoctacyclo $\left[12.12 .2 .1^{26}, .0^{7}, 28.0^{8}, 13.0^{15}, 19.0^{20}, 27.0^{21},{ }^{26}\right]$ nonacosa$8,10,12,14,19,21,23,25,27-n o n a e n-16-$ one

(7S,9S)-7-[(2R,4S,5S,6S)-4-amino-5-hydroxy-6-methyloxan-2-yl] oxy-6,9,11trihydroxy-9-(2-hydroxyacetyl)-4-methoxy-8,10-dihydro-7H-tetracene-5,12dione

(2R,6aS, 12aS)-1,2,6,6a,12,12a-hexahydro-2-isopropenyl-8,9dimethoxychromeno[3,4-b]furo(2,3-h)chromen-6-one

1-(3-lodophenyl)-4-(3-nitrophenyl)-1H-1,2,3-triazole

Methyl(1R,9R,10S,11R,12R,19R)-11-(acetyloxy)-12-ethyl-4-[(13S,15S,17S)-17ethyl-17-hydroxy-13-(methoxycarbonyl)-1,11-diazatetracyc lo[13.3.1.0 ${ }^{4}, 12.0^{5},{ }^{10}$ ]nonadeca-4(12),5,7,9-tetraen-13-yl]-8-formyl-10-hydroxy5-methoxy-8,16-diazapentacyclo[10.6.1.0 $\left.{ }^{1},{ }^{9} \cdot 0^{2},{ }^{7} \cdot 0^{16},{ }^{19}\right]$ nonadeca-2,4,6,13tetraene-10-carboxylate

(2-\{4-[(1Z)-1,2-diphenylbut-1-en-1-yl] phenoxy\}ethyl)dimethylamine
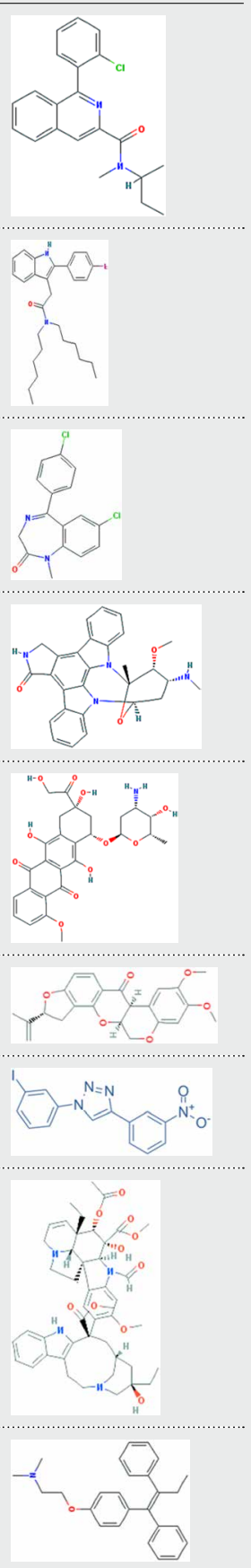

Ultrastructural imaging analysis of MCF-7 cells via transmission electron micrography (TEM) pictured the disrupted cristae and reduced mitochondrial-nuclear distance (passing from $\sim 120$ to almost $\sim 50 \mathrm{~nm}$ in response to STS) (Fig. 3, G and H). This observation prompted us to investigate the mechanisms dictating such a relevant gain in space between mitochondria and nucleus during apoptosis.

\section{Mitophagy-evading mitochondria, rich in TSPO, form contact sites with the nucleus during MRR}

In MCF-7 and MDA cells, mitochondria and nuclear envelope were stained for TSPO and Lamin B, respectively. This analysis showed that in MDA cells, infiltrates of TSPO in the perinuclear region establish a constitutive space occupancy by mitochondria, which is indicative of physical interaction (Fig. 4, A and B). This interconnection 
A

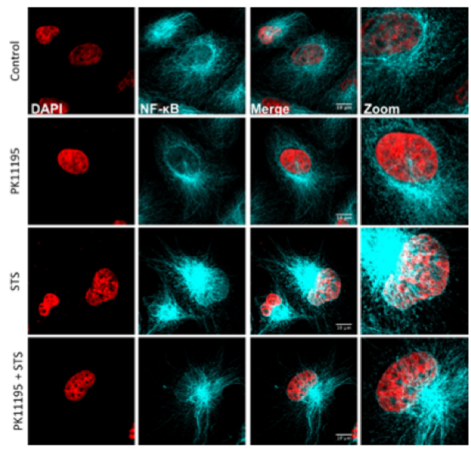

D

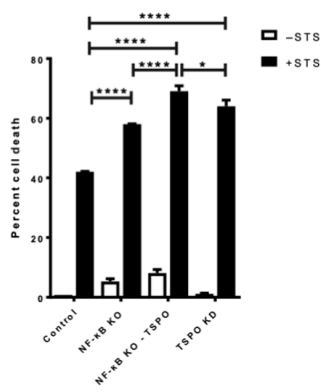

B

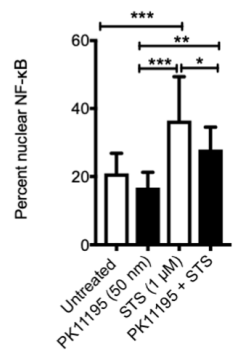

E

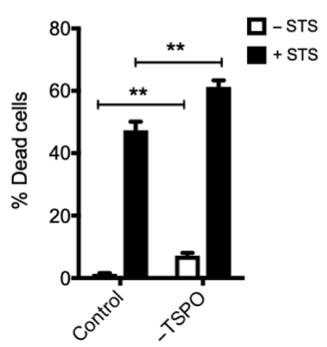

C

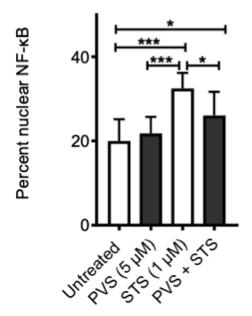

F

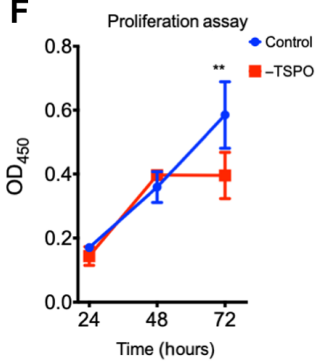

G

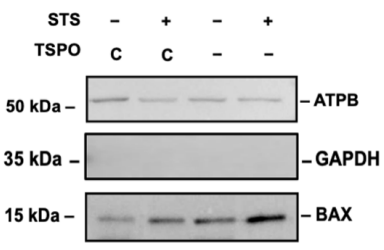

K

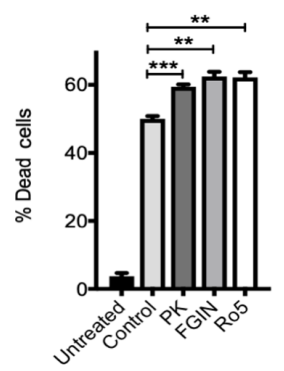

H

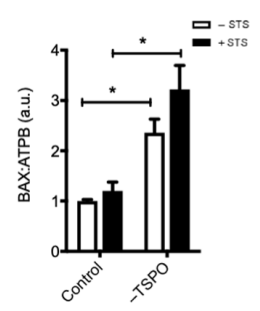

I

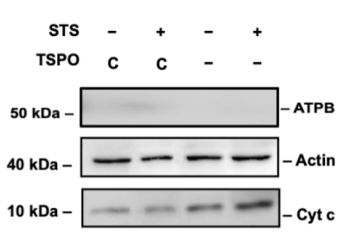

J

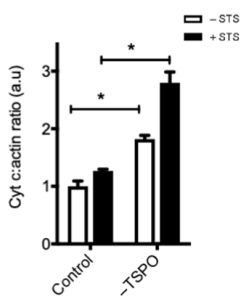

L

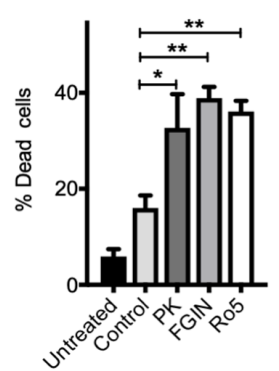

M

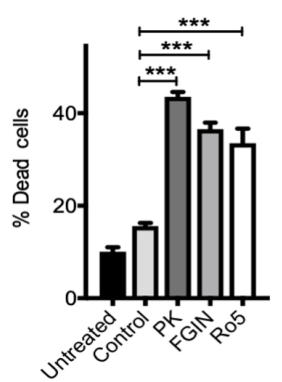

Fig. 2. Molecular and pharmacological modulation of TSPO dictate the degree of NF-kB retro-translocation and mitochondrial commitment to apoptosis. (A) Immunocytochemical analysis of nuclear NF-KB (cyan) in MDA cells untreated or treated with STS in the absence or presence of the TSPO ligand PK11195. NF- $\mathrm{kB}$ signal is quantified in (B) (control: $26.86 \pm 15.02$; PK11195: $21.26 \pm 12.24$; STS: $49.36 \pm 23.52 ;$ PK11195 + STS: $34.52 \pm 21.36 ; n=15)$, and (C) reports the evaluation of nuclear NF-KB signal following STS treatment in combination with pravastatin (PVS) (control: $25.14 \pm 14.78$; PVS: $25.70 \pm 17.88$; STS: $36.20 \pm 26.64$; PVS + STS: 32.59 $\pm 21.00 ; n=10$ ). Histo-

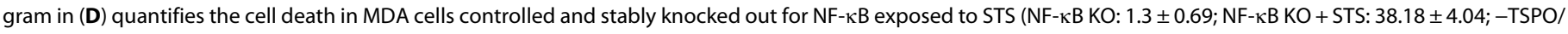
NF-кB KO: $2.88 \pm 0.07 ;-T S P O / N F-\kappa B ~ K O+S T S: 37.1 \pm 3.45 ; n=3$ ), while (E) refers to the TUNEL (terminal deoxynucleotidyl transferase-mediated deoxyuridine triphosphate nick end labeling) assay in control and -TSPO MDA cells exposed to STS (control: $1.37 \pm .27$; STS: $47.38 \pm 2.74 ;-T S P O: 7.19 \pm 0.85 ;-T S P O+$ STS: $61.25 \pm 2.06 ; n=3$ ). (F) Recording of the proliferation assay in the same cells. The Western blot in (G) shows the higher degree of Bax accumulation in mitochondrial fractions of -TSPO MDA cells following STS treatment quantified in the plot of $(\mathbf{H})$ (control: 1 ; STS: $1.2 \pm 0.18 ;-T S P O: 2.3 \pm 0.27 ;-T S P O+$ STS: $3.1 \pm .048 ; n=3$ ). The Western blot in (I) is the analysis of Cyt c levels in the cytosolic portion, and (J) is its quantification (control: 1 ; control + STS 1.82 $\pm .06 ;-T S P O: 1.27 \pm 0.03 ;-T S P O+$ STS: $2.79 \pm 0.18 ; n=3$ ). The exacerbation of STS-induced cell death by TSPO ligands PK11195, FGIN, and Ro-5 is reported in (K), (control: $3.71 \pm 0.94$; STS: 49.98 \pm 0.84 ; STS + PK11195: 59.43 \pm 0.68 ; STS + FGIN: $62.41 \pm 1.4 ;$ STS + Ro-5: $62.16 \pm 1.57 ; n=3$ ). Ligands that coadjuvate also doxorubicin-induced cell death (L) (control: $5.9 \pm 1.52$; doxorubicin: $16.00 \pm 2.63 ;$ doxorubicin + PK1 1195: 32.7 \pm 7.03; doxorubicin + FGIN: $38.9 \pm 2.3$; doxorubicin + Ro-5: $36.1 \pm 2.23 ; n=4$ ) and vincristine $(\mathbf{M})$ (control: $10.05 \pm 0.95 ;$ vincristine: $15.61 \pm 0.67$; vincristine + PK11195: $43.6 \pm 1.02$; vincristine + FGIN: $36.54 \pm 1.41$; vincristine + Ro-5: $33.53 \pm 3.13 ; n=3) .{ }^{*} P \leq 0.05 ;{ }^{* *} P \leq 0.01 ; * * * P \leq 0.001$. 
A

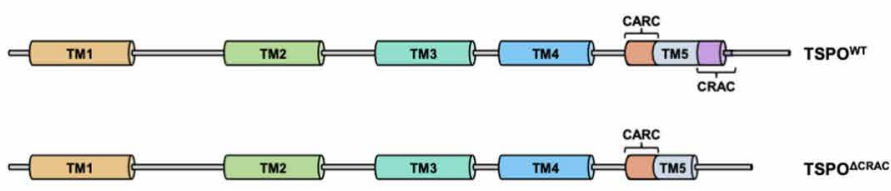

C

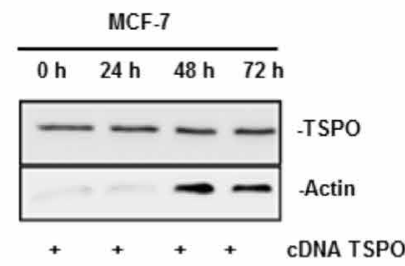

F
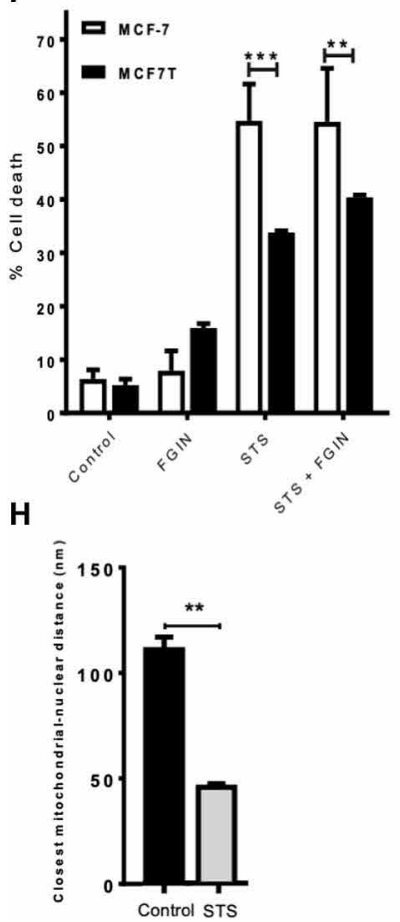

D

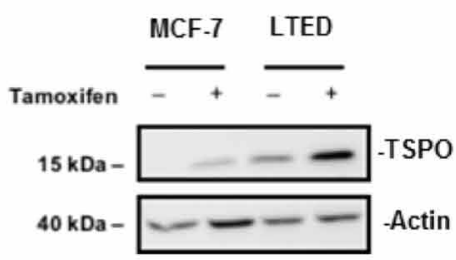

G
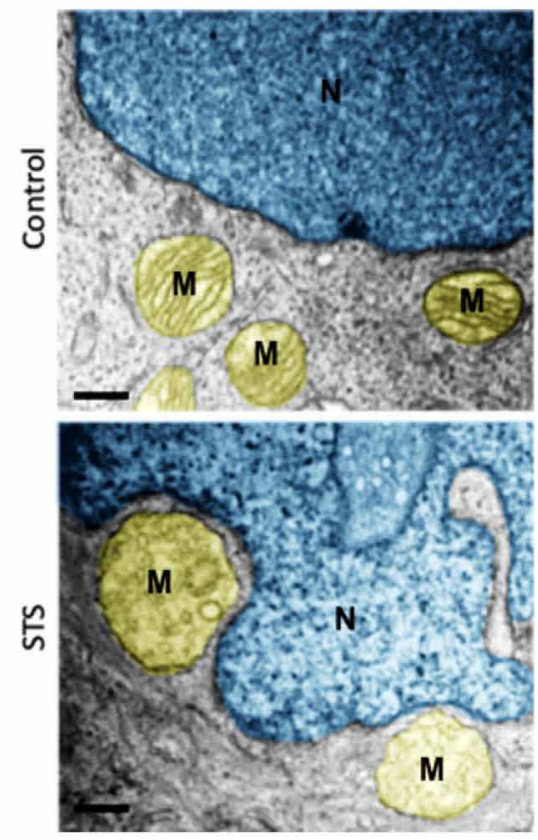

B
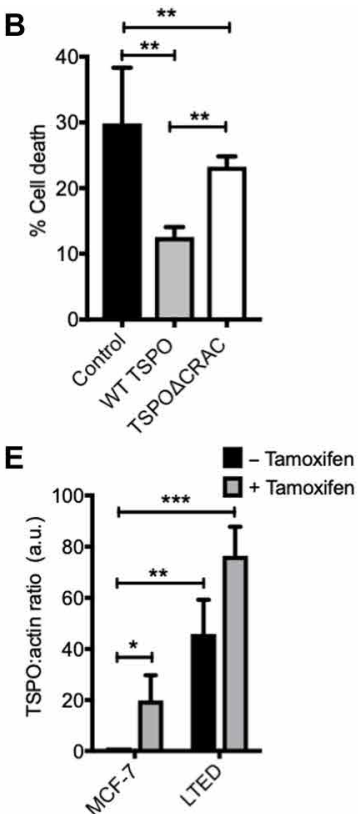

Fig. 3. Cholesterol-binding capacity is pivotal for TSPO-mediated prevention of programmed cellular demise and mitochondrial associated with the nucleus. The domain model in (A) refers to the WT TSPO and TSPO $\triangle$ CRAC constructs expressed into MCF-7 cells whose degree of STS-induced cell death was quantified at 16-hour treatment (B) (control: $29.86 \pm 8.48 ;+$ TSPO: $15.37 \pm 4.6 ;+$ TSPO $\triangle$ CRAC: $2.21 ; n=5$ ) at the time expression of plasmids was maximal: 48 hours as shown in (C). The long-term estrogen-deprived (LTED) MCF-7 cells, which are more resistant to cell death, express higher level of TSPO, which is enhanced further by tamoxifen (D). The significant differences in TSPO accumulation with and without tamoxifen are quantified in (E) (MCF-7 control: 1; MCF-7 + TAM: 19.85 \pm 9.8; LTED control: $45.88 \pm 13.3$; LTED + TAM: $76.41 \pm 11.3 ; n=3$ ). (F) Cell death analysis in the same cells showing that MCF7T cells are resistant to STS in comparison to control MCF-7 (MCF-7 control: $8.09 \pm 4.68$, MCF7T control: $7.17 \pm 3.34$, MCF-7 + FGIN: $11.65 \pm 4.22$, MCFT + FGIN: $17.33 \pm 14.58$, MCF-7 + STS: $61.61 \pm 47.89$, MCF7T + STS: $34.38 \pm 33.31$, MCF-7 + FGIN + STS: $64.56 \pm 44.49$, MCF7T + FGIN + STS: $41.11 \pm 39.71 ; n=3)$. The transmission electron micrography (TEM) presented in (G) shows the remodeling of the mitochondrial network (M; yellow) toward the nucleus ( $\mathrm{N}$; blue) in STS-treated MCF-7 cells. The quantification reported in $(\mathbf{H})$ indicates the gain in distance (greater than $50 \mathrm{~nm}$ ) between mitochondria and the nucleus after STS $(n=5) .{ }^{*} P \leq 0.05 ;{ }^{* *} P \leq 0.01 ;{ }^{* * *} P \leq 0.001$. 


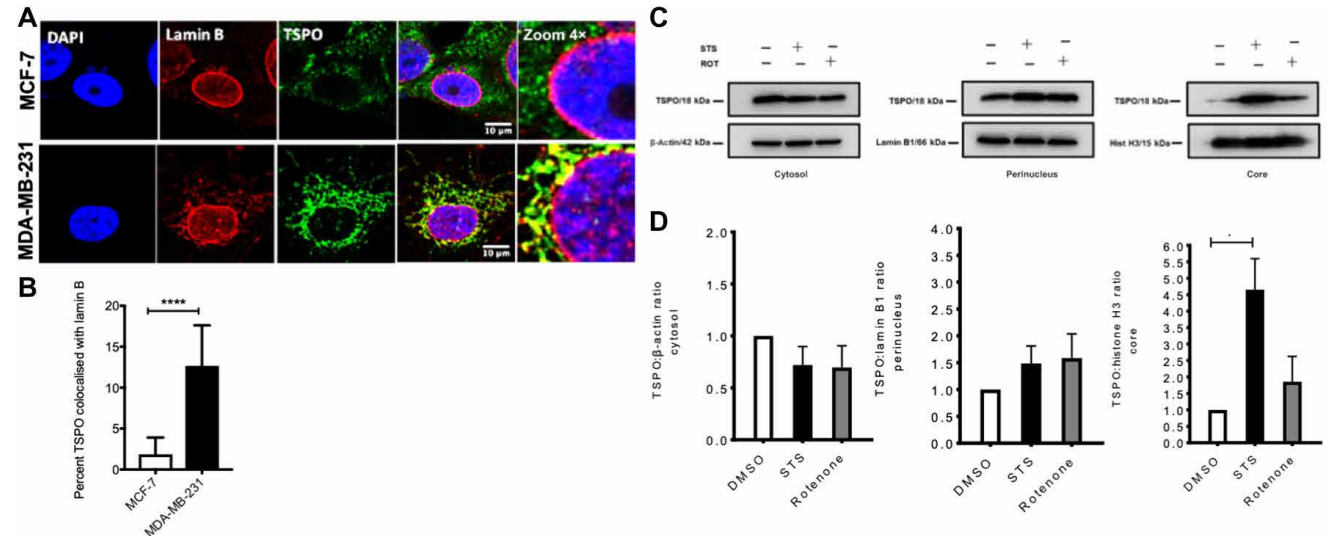

E

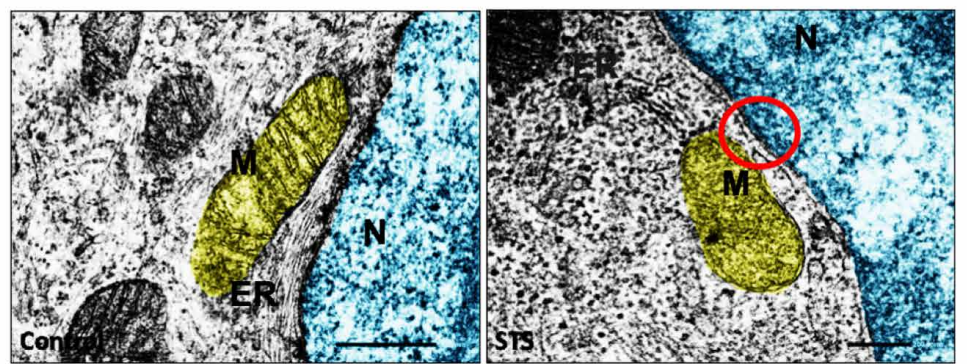

$\mathbf{F}$
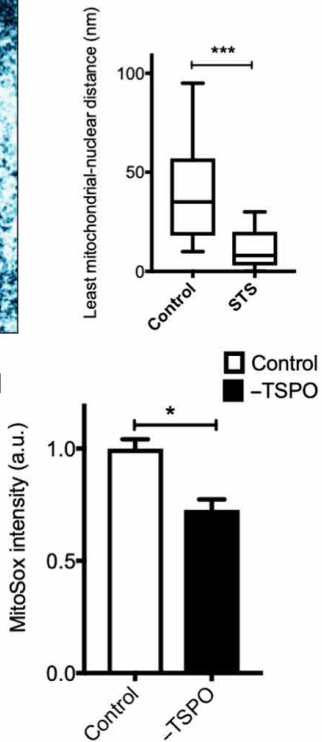

J
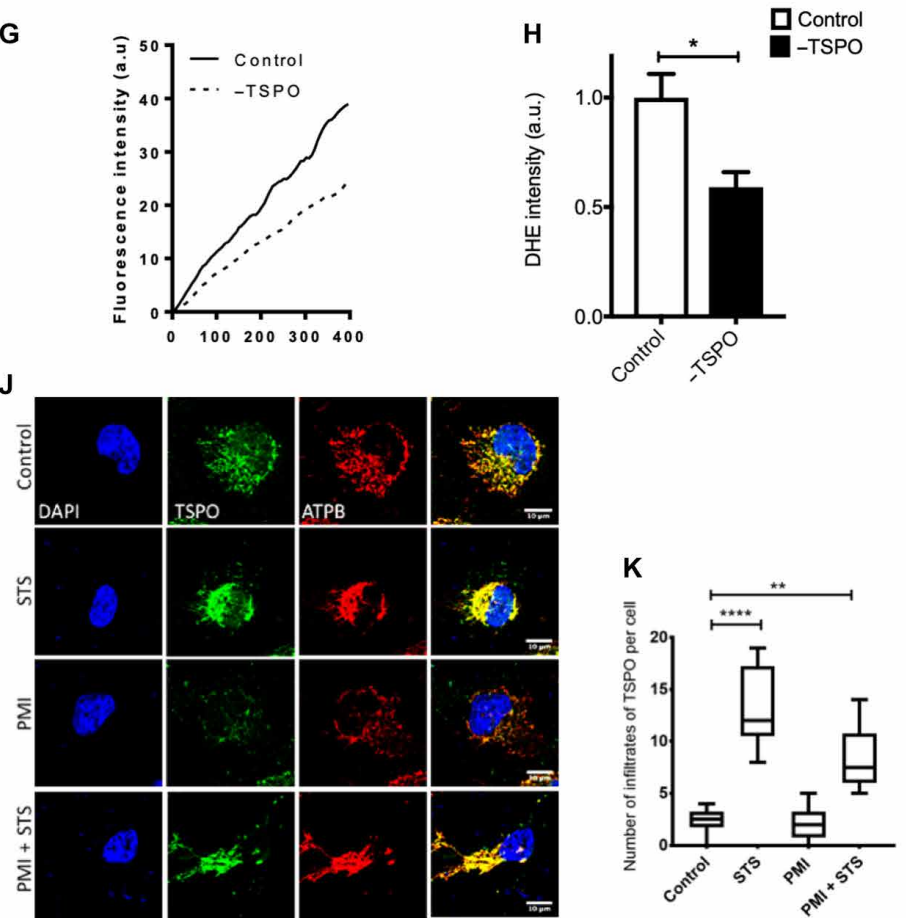

$\mathbf{L}$

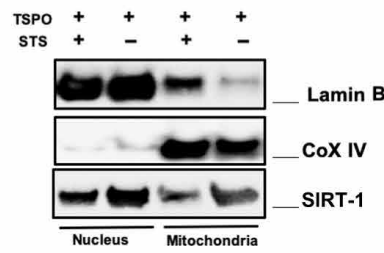

Fig. 4. Nucleus-associated mitochondria (NAM) form during induction of MRR, for which the inhibition of mitophagy is required. The immunocytochemical analyses in (A) investigate the proximity of the mitochondrial network to the nucleus in MCF-7 and MDA cells with relative quantification in (B) (MCF-7: $1 \pm 0.9 ;$ MDA cells: $5.40 \pm 1.5 ; n=3)$. The Western blot in (C) shows the redistribution of TSPO in the nucleus following treatment with STS and rotenone (ROT) with densitometry reported in (D) (TSPO: $\beta$-actin ratio in the cytosol, control: 1; STS: $0.89 \pm 0.54$; ROT: $0.905 \pm 0.48 ; n=3$; TSPO:lamin B1 ratio in the perinucleus, control: 1; STS: $1.81 \pm 1.16$; ROT: $2.03 \pm 1.13 ; n=3$; TSPO:histone H3 ratio in the nuclear core, control: 1; STS: $5.60 \pm 3.70$; ROT: $2.62 \pm 1.08 ; n=3$ ). (E) TEM micrographs of dimethyl sulfoxide (control) and STS-treated MDA cells confirm the physical interaction between mitochondria (M; yellow) and the nucleus ( $\mathrm{N}$; blue). (F) Quantification of the distance between mitochondria and the nucleus in control and STS-treated MDA cells (control: $65.45 \pm 13.89 \mathrm{~nm} ; \mathrm{STS}: 21.75 \pm 1.19 \mathrm{~nm} ; n=15$ ). (G) Kinetics of dihydroethidium (DHE)-based measurement of ROS production in control and -TSPO MDA cells. The quantification in (H) confirms the lower cytoplasmic ROS in -TSPO cells (control: $1 ;-T S P O: 0.59 \pm 0.07 ; n=20$ ). The average mitochondrial accumulation of the DHE derivative MitoSox (I) reports significantly low ROS levels in -TSPO MDA cells (control: $1 ;-T S P O: 0.73 \pm 0.04 ; n=20$ ). (J) Confocal imaging of MDA cells exposed to STS, PMI, or STS + PMI: The latter prevents remodeling of the mitochondrial network on the nucleus $(n=5)$. The box plot in $(\mathbf{K})$ reports the quantification of infiltrates per cell (STS: $16.17 \pm 11.17 ;$ STS + PMl: $10.67 \pm 6.98 \mu \mathrm{m}$; $n=9$ ). Last, the Western blot in (L) shows the accumulation of the nuclear protein lamin $B$ on the mitochondria during STS treatment. ${ }^{*} P \leq 0.05 ;{ }^{* *} P \leq 0.01 ; * * * P \leq 0.001$. 
increases in response to STS, as detailed by both immunoblotting of subcellular fractions (Fig. 4, C and D) and TEM analyses (fig. S2, A and B). TSPO, in response to STS, was observed to be present in the portion of the cytosol occupied by mitochondria as well as in the nuclear envelope and its core (Fig. 4, C and D). A similar dynamic redistribution of mitochondria (read by the accumulation of TSPO) is obtainable also by the mitotoxin rotenone (ROT) (43), which induces MRR (Fig. 4, C and D).

The ultrastructural analysis also revealed the prominent interorganellar coupling that generated an all-encompassing structure in and around the nucleus, which we called the nucleus-associated mitochondria (NAM). Means of quantification revealed the formation of actual contact sites with a statistical reduction in distance-space between mitochondria and nucleus below $30 \mathrm{~nm}$ and observable membrane fusion (Fig. 4, E and F).

The mitochondrial remodeling could therefore crucially influence the signaling of diffusible molecules such as ROS, which are acknowledged factors in the retrograde response $(1,14)$ and dependent on their tonicity by the level of TSPO (16). In line with this, both cytoplasmic and mitochondrial ROS levels decrease when TSPO is down-regulated (Fig. 4, G to I). The NAM-induced change in spacedistance would therefore favor the diffusion capacity of ROS, maximizing their impact on perinuclear and nuclear regions. Notably, the remodeling of mitochondria on the nucleus, alike the retro-translocation of NF- $\kappa \mathrm{B}$, is limited by the mitophagy-driven trimming of the network, here induced by PMI (Fig. 4, J and K) (31). The formation of NAM was further corroborated by assessing the level of lamin B in purified mitochondrial fractions of cells exposed to STS treatment (Fig. 4l).

\section{A TSPO-mediated molecular complex (TSPO-ACDB3-PKA- AKAP95) tethers mitochondria to the nucleus}

NAM form also in the mouse embryonic fibroblasts (MEFs), in which the expression level of TSPO is equally critical as in cancer cells to establish mitonuclear contacts (Fig. 5, C to F). "Variance" filter applied to confocal Airyscan images with immunofluorescence [lamin B1-ATP synthase (ATPB)] convincingly detail how the downregulation of TSPO (-TSPO) in MDA cells releases mitochondria from the nucleus, while its overexpression (+TSPO) in MEFs results in the coalescing of the mitochondrial network with the nuclear envelope (Fig. 5, A to D). These observations were corroborated at ultrastructural level via TEM analysis, which unequivocally showed the formation of contacts between mitochondria and the nucleus in the face of increased TSPO expression (Fig. 5, E and F). TSPO-overexpressing MEFs (which feature accumulated NF- $\kappa \mathrm{B}$ in their nucleus) gained significant resistance to STS-induced cell death, which was otherwise lost when the $\triangle \mathrm{TSPO}$-CRAC mutant clone of the protein (unable to bind cholesterol) was expressed (Fig. 5G).

We then attempted to dissect the mechanisms by which TSPO could mediate such a tethering role between mitochondria and the nucleus, hypothesizing a partnering activity with other molecules. Bioinformatic analysis outlined the TSPO interactors (Fig. $5 \mathrm{H}$ ), and among these, we teased out those with established anchoring capacity and capable of an alternative intracellular location from the mitochondria. From the analysis, ACBD3, which is a multifunctional interactor of TSPO and PKA $(23,24)$, emerged as a main candidate. Considering that PKA, under stress, is recruited on the nucleus and anchored via AKAP95 $(25,26)$, we therefore hypothesized the formation of a multiprotein complex formed by TSPO, ACBD3, PKA, and AKAP95, which tethers mitochondria to the nucleus (Fig. 5I).
This is far more evident under STS-induced stress, when PKA pools on the nucleus increase in MDA cells (Fig. 5, J to L). To further corroborate this hypothesis, we employed coimmunoprecipitation analyses to determine whether TSPO and AKAP95 interact. When the expression of TSPO is high, as in the MDA, the interaction with AKAP95 is evident and ACBD3 is mediated (Fig. 5, M and N). Knocking down ACBD3 (-ACBD3) (transfection efficiency shown in fig. S4J) is instead sufficient to impair this interaction, thereby reducing the association between mitochondria and the nucleus (fig. S5, A and B).

The distinctive molecular nature of NAM from the mitochondriaassociated ER membranes (MAM) was also proven by assessing their level in cells devoid of the integral membrane protein VAPB5 and mitofusin 2 (44), whose removal did not affect the connection between mitochondria and the nucleus (figs. S4, F and G, and S5, C and D).

\section{NAM exploit TSPO to redistribute cholesterol into the nucleus and promote NF-кB-mediated transcription of genes}

Oxidized forms of cholesterol as demonstrated by the use of the oxysterol 7 -ketocholesterol $(7-\mathrm{KC})(45,46)$ promote the accumulation of NF- $\kappa \mathrm{B}$, and subsequently the formation of NAM (Fig. 6, A to E).

Notably, this treatment modifies the pattern of TSPO expression too as well as that of other components of the steroidogenic protein complex transduceome (19): the oxysterol receptor liver X receptor $\beta$ (fig. S2, F and G) (47), the ATPase (adenosine triphosphatase) family AAA domain-containing protein 3 (ATAD3) (fig. S2, H and I), and the steroidogenic acute regulatory protein (STAR) (fig. S2, H and J). This, in turn, suggests a mechanistic interplay between the mitochondrial handling of cholesterol, its metabolism, and MRR. Even though other lipids may be involved in this route of communication, we detailed the redistribution of cholesterol after remodeling of the mitochondrial network during MRR. In MDA cells expressing the red variant of the green fluorescent protein (mt-RFP), we imaged the pools of cholesterol via the fluorescent compound filipin, which depicted the localization of the lipid into foci close to and around the nucleus: foci that disappeared in -TSPO MDA cells (Fig. 6, F and G).

The STS-induced retro-trafficking of cholesterol was confirmed by imaging the lipid enrichment of the nuclear membranes in MDA cells with the fluorescent probe Di-4-ANEPPDHQ (Fig. 6, H and I). In the same conditions, the efflux of cholesterol from the nucleus could be observed during cell treatment with TSPO ligands (Fig. 6J), as well its marked influx when the protein is overexpressed (Fig. 6K). The pattern of cholesterol was also assessed by live imaging using dehydroergosterol [ergosta-5,7,9(11),22-tetraen-3 $\beta$-ol, DHE] (48), which showed the inhibition of this nuclear redistribution of the lipid when cells are cotreated with the TSPO ligand PK11195 (49) or the intracellular cholesterol transport inhibitor U1866A (Fig. 6, L and M). Most notably, the pharmacological targeting of TSPO with PK11195 did also prevent remodeling of mitochondria (Fig. 6, $\mathrm{N}$ and $\mathrm{O}$ ).

The interdependence between cholesterol accumulation in the nucleus and transcriptional activity of NF- $\mathrm{KB}$ was also confirmed by monitoring the expression levels of the prosurvival genes (c-FLIP and Bcl-2), both of which were reduced by the cholesterol synthesis inhibitor lovastatin (Fig. 6, P and Q), likewise in -TSPO cells (Fig. 1, N and O). Furthermore, we gained evidence of the increased levels of NF- $\kappa \mathrm{B}$ deacetylation, which is indicative of its activation, during STS treatment, associated with the reduced nuclear accumulation of the nutrient-sensing deacetylase Sirtuin-1 (SIRT-1) $(50,51)$, an 

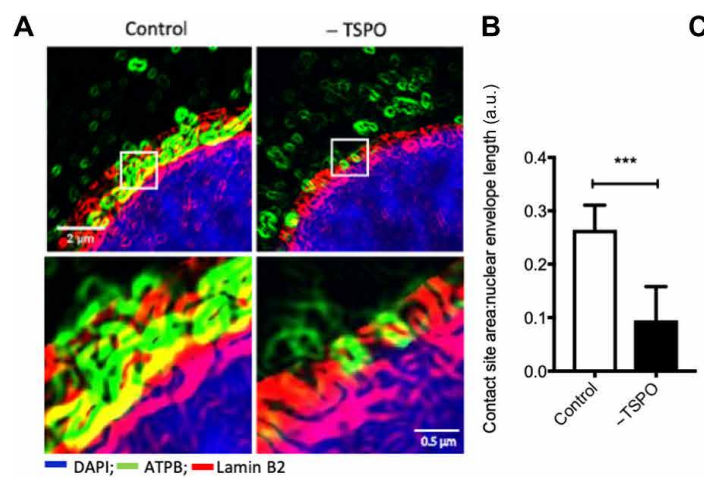

E

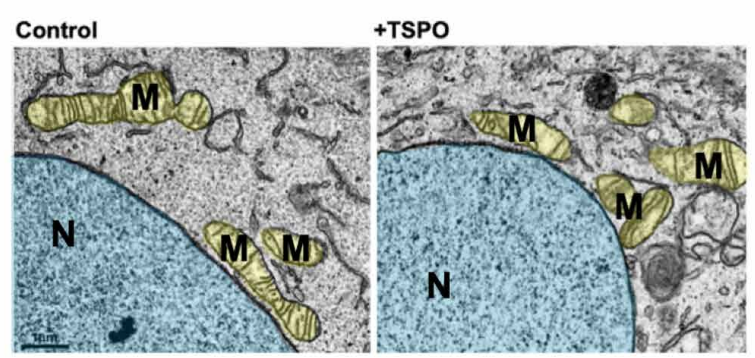

H
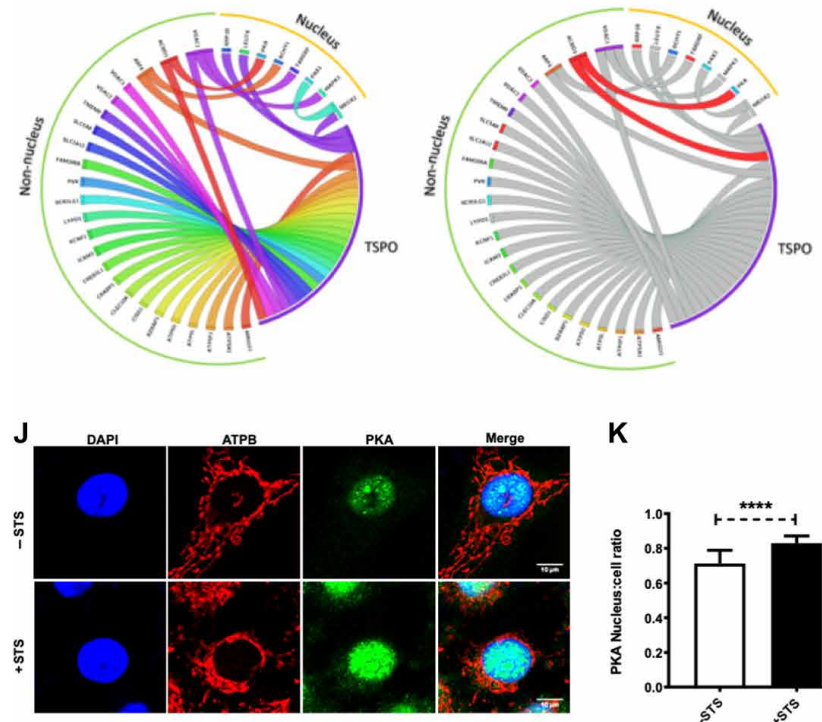

$\mathbf{K}$

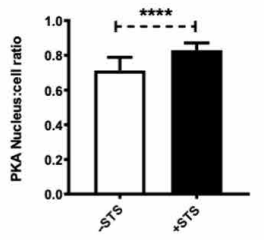

F

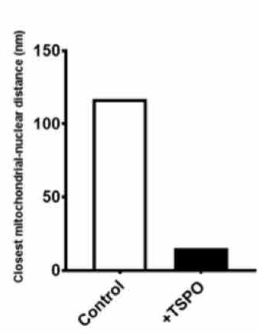

G

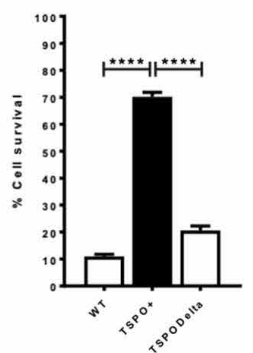

I

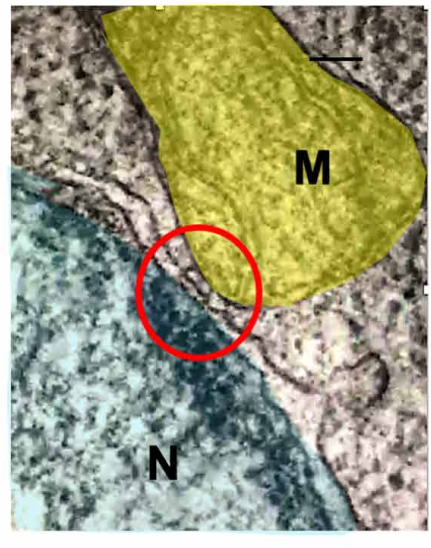

$\mathbf{L}$

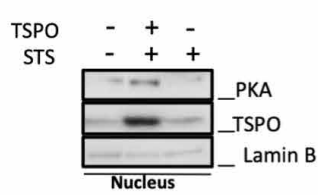

"

\begin{tabular}{|c|c|c|c|}
\hline Input & IP & FT & IP: AKAP95 \\
\hline & -2 & & WB: AKAP95 \\
\hline
\end{tabular}

$\mathbf{N}$

\begin{tabular}{|c|c|c|c|c|c|c|c|}
\hline \multirow[b]{2}{*}{ ACBD3: } & \multicolumn{2}{|c|}{ Input } & \multicolumn{2}{|c|}{ IP } & \multicolumn{2}{|c|}{ FT } & \multirow{2}{*}{ IP: AKAP95 } \\
\hline & WT & KD & WT & KD & WT & KD & \\
\hline & $=$ & $=$ & $=$ & $=$ & & & WB: AKAP95 \\
\hline & & & $=0$ & & & & WB: TSPO \\
\hline
\end{tabular}

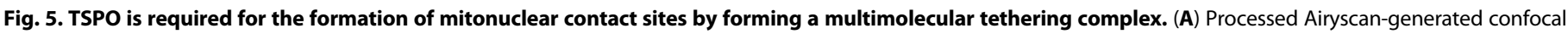

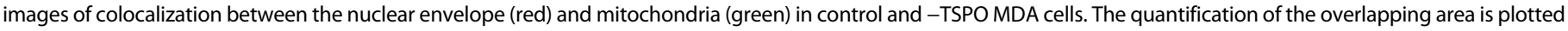

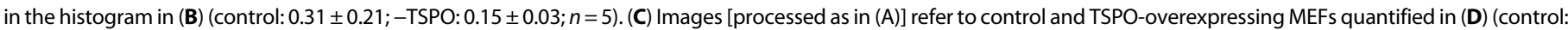

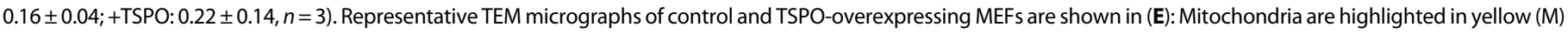

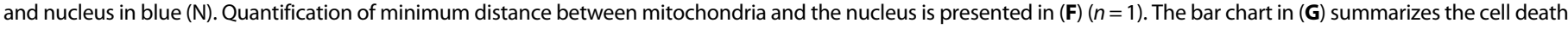

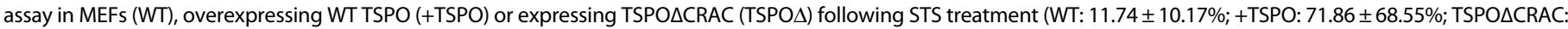

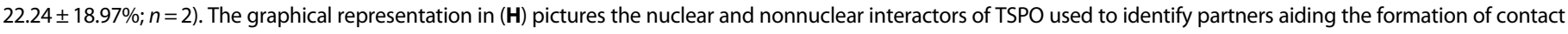

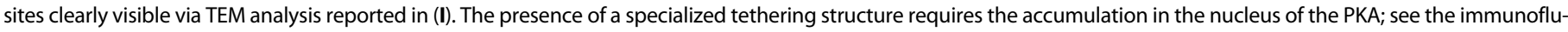

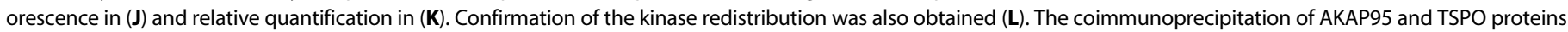

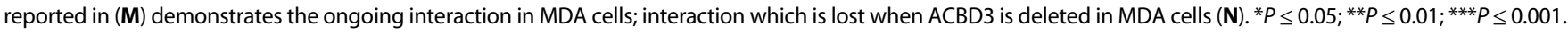


A
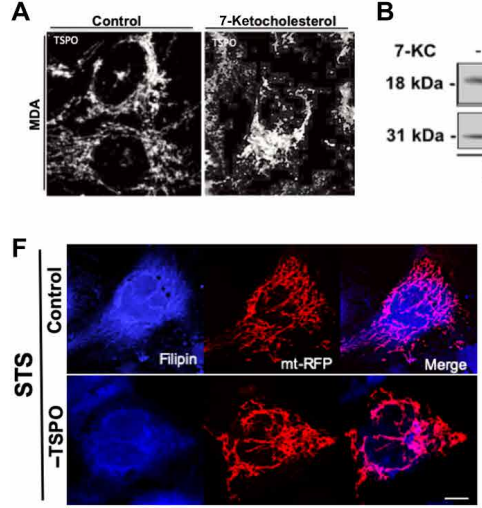
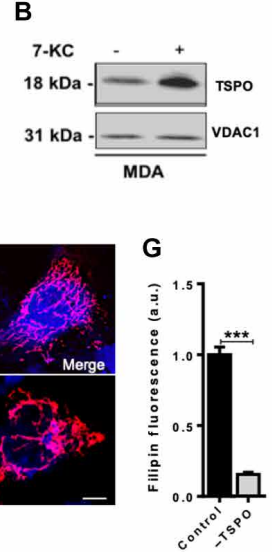

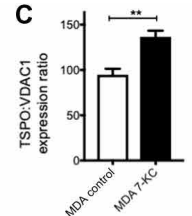

D

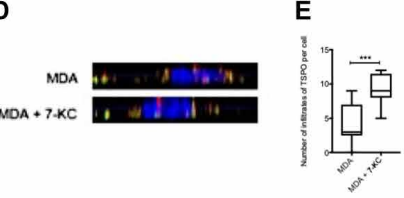

$\mathrm{H}$

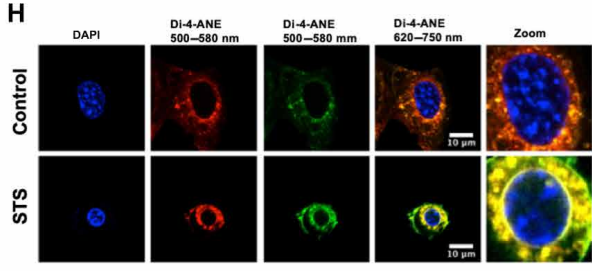

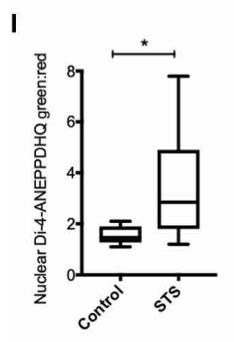

$$
\mathbf{J}
$$

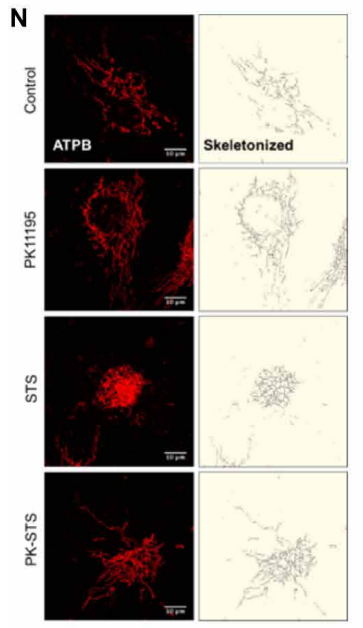

$\mathbf{R}$

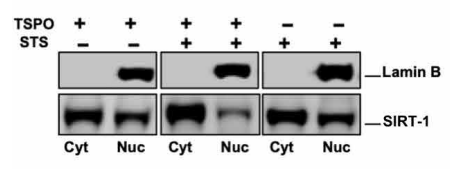

K

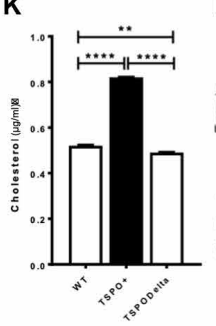

L

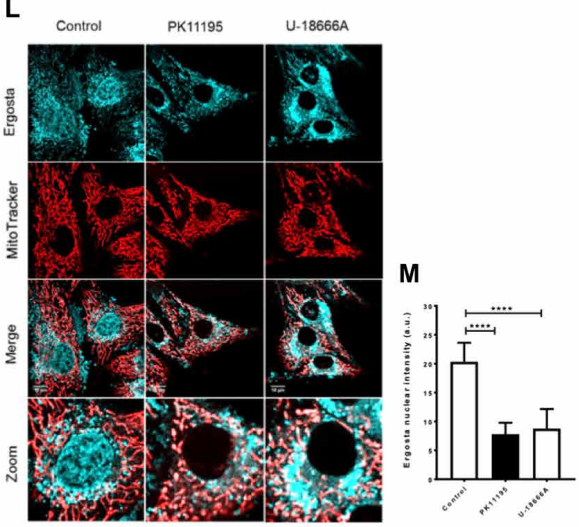

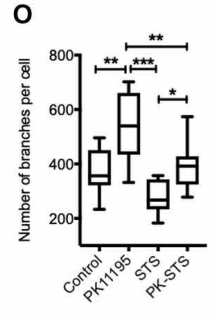

S

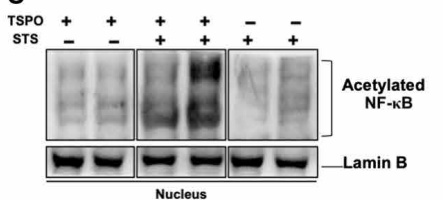

Q
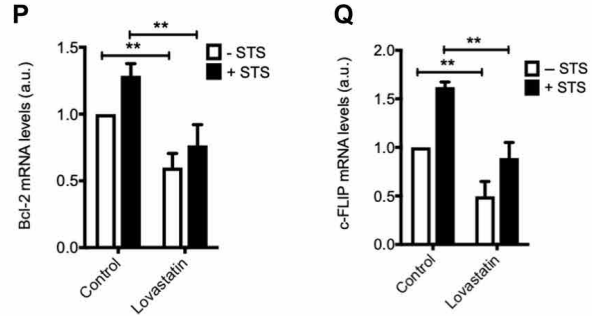

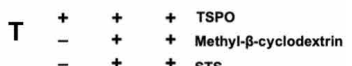

STS

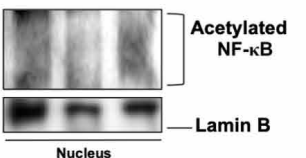

Fig. 6. Redistribution of cholesterol in the nucleus aids NF- $\mathrm{kB}$ activity is secondary to NAM formation and requires functional TSPO. Immunostained MDA cells incubated with 7-KC show TSPO up-regulation (A) confirmed by protein analyses in (B) and quantified in (C) (control: 94.86 $\pm 6.5,7-K C: 136.59 \pm 6.76 ; n=3$ ). Orthogonal projection of the mitochondrial infiltrates in response to $7-\mathrm{KC}$ is pictured in (D) with numbers plotted in (E) (control: 4.22 $\pm 2.9 ; 7-\mathrm{KC}: 12.34 \pm 2.3 ; n=9$ ). Confocal images of - TSPO MDA cells stained with the cholesterol marker filipin (F) reveal the amount of nuclear accumulation in the conditions plotted in (G) (control: $1.04 \pm 0.009 ;-T S P O$ : $0.2 \pm 0.001 ; n=8$ ). (H) Cholesterol stained with the dye Di-4-ANEPPHQ (ANE), and the plot in (I) reports its accumulation in the nuclear envelope following STS. The bar chart in $(J)$ is the analysis of nuclear cholesterol $(\mu \mathrm{g} / \mathrm{ml})$ in MDA cells treated with PK11195 and Ro-5 (control: 1; PK11195: 0.86 $\pm 0.008 ;$ Ro-5: 0.54 $\pm 0.03 ; n=3$ ); nuclear cholesterol was also quantified in MEFs overexpressing TSPO (+TSPO) or expressing its mutated isoform (TSPO $\Delta$ ) (K). Screen shots in (L) are of the live-cell imaging analysis of the cholesterol analog ergosta-5,7,9(11),22-tetraen-3ß-ol in CF35 cells costained with MitoTracker Red with data plotted in (M) (control: 23.629 \pm 17.03 ; PK11195: $9.78 \pm 5.81 ; \mathrm{U}-18666 \mathrm{~A}: 12.16 \pm 5.36 ; n=25)$. Confocal images in $(\mathbf{N})$ indicate that PK11195 prevents remodeling of mitochondria as confirmed by the plot of the corresponding skeletonized mitochondrial network in (0) (control: 371.6 \pm 2.3 ; PK11195: $532 \pm 12$; STS: $275 \pm 62.9$; PK11195 + STS: $390 \pm 86.5 ; n=10$ ). (P and Q) Analysis of Bcl-2 and c-FLIP mRNA levels in cotreatment with STS and lovastatin (Bcl-2, control: 1; STS: $1.08 \pm 0.04$; vehicle: $0.6 \pm 0.18$; lovastatin + STS: $0.77 \pm 0.2 ; n=3$; c-FLIP, control: 1 ; STS: $1.49 \pm 0.32$; lovastatin: $0.052 \pm 0.1$; lovastatin + STS: $0.85 \pm 0.10 ; n=3$ ). Last, the protein analysis of Sirtuin- 1 in the nucleus is reported in $(\mathbf{R})$ and that of NF- $K B$ deacetylation in (S) and (T) in response to TSPO repression and/or methyl- $\beta$-cyclodextrin treatment $(n=6)$. ${ }^{*} P \leq 0.05 ;{ }^{* *} P \leq 0.01 ;{ }^{* * *} P \leq 0.001$. 


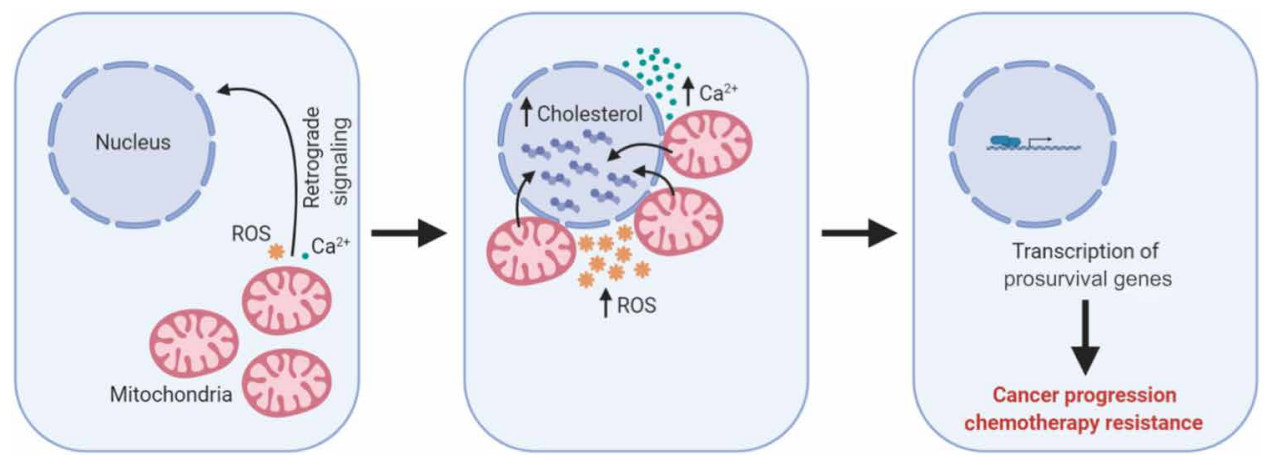

Fig. 7. Working model of the formation of contact sites between mitochondria and the nucleus (NAM) during the execution of the MRR. The juxtaposition of mitochondrial and nuclear membranes facilitates the convergence of mitochondria-derived signaling molecules to the nucleus, activating prosurvival pathways such as the one controlled by NF-kB. The redistribution of cholesterol from the mitochondrial compartment to the nucleus takes place during the MRR process in which ROS and $\mathrm{Ca}^{2+}$ take part.

event that is favored by the repression of TSPO level (Fig. 6, R and S). Depletion of cellular cholesterol via methyl- $\beta$-cyclodextrin mimics similar protective effect on deacetylation of the NF- $\mathrm{\kappa B}$ (Fig. 6T).

The final graphical model in Fig. 7 summarizes the key steps of this pathway, in which association of mitochondria with the nucleus and redistribution of cholesterol regulate MRR activation and execution along with alterations in $\mathrm{Ca}^{2+}$ and ROS signaling.

\section{DISCUSSION}

In this work, we demonstrate that mitochondria can establish points of contact with the nucleus to favor spatially confined biochemical events that promote nuclear stabilization of prosurvival transcriptional factors such as NF- $\kappa$ B. We called these newly identified contact sites NAM. We also report that cholesterol works as an intermediate or a facilitator of the mitochondria-to-nucleus retro-communication together with ROS and $\mathrm{Ca}^{2+}$, which have been acknowledged as the prevalent dynamic signaling molecules between the two organelles $(1,2)$. The accumulation of cholesterol in the nuclear envelope emerges as regulator of cellular reprogramming toward stress resistance and survival (Fig. 6, F to M). Even though cholesterol is known to affect transcription of genes (47), it is here reported to be trafficked into hot spots at the interface between mitochondria and the nucleus to promote their communication, thus resembling other platforms for the exchange of information such as those between the ER and mitochondria (ER-MAMs) (52), which we prove to be molecularly distinct from the NAM (fig. S5, F and G).

Following mitochondrial reorganization, TSPO may therefore deliver its lipid cargo, cholesterol, into the nuclear core of the cell where the concurrence of ROS may lead to auto-oxidative products of cholesterols (oxysterols) (46). The pattern of elements of the transduceome complex (31), which all converge into the nucleus along with TSPO, strongly argues for this (fig. S2, E to J). We speculate that the enrichment of the nuclear envelope with lipids such as cholesterol, inducing a change in the physical properties (Fig. 6H), represents a crucial step in the altered transcriptional profile that enables aggressive cancer cells to differentiate from their less persistent counterparts. Here, we unveiled that cholesterol is pivotal for the efficiency of the retrograde response as the degree of NF- $\mathrm{KB}$ acetylation is affected by its redistribution in the nucleus (Fig. 6, $\mathrm{R}$ to $\mathrm{T}$ ), which represses the NAD-dependent deacetylase SIRT- 1 as previously proposed $(50,51)$.
While the observation that the mitochondrial interactome is crucial to cellular health is not new (53), the fine regulation of mitochondrial juxtaposition on the nucleus is. We have here detailed that TSPO is required for this interorganellar interaction, and, in turn, its ligands work as efficient pharmacological modulators of this coupling (Fig. 6, L and M). We propose that a pool of proteins formed by ACBD3, PKA, and AKAP95 bridge TSPO-positive mitochondria and the nucleus: A tether that is visible in the tomograph reported in Fig. 5I and fig. S5E. Even though we cannot rule out the possibility that a voltage-dependent anion channel (VDAC) is required to ensure the right positioning of the OMM and tethering with the nucleus, we envisage a relay between stress proteins such as TSPO, ACBD3, and PKA $(23,24)$.

TSPO is an ancient molecule (54), and the part it plays in the NAM may be an evolutionary adaptation to bridge the two genomes. Clearly, it is exploited in such a scope during pathologies.

NAM are prominent in the aggressive line (MDA) derived from advanced lesions of the mammary gland when compared with the less aggressive counterpart: the MCF-7. In this cellular model, STS treatment is nonetheless able to promote a statistically significant reduction in the distance between mitochondria and the nucleus (Fig. 3, G and $\mathrm{H}$ ), even though with reduced capacity than in MDA cells (Fig. 4, A and B). This steady-state formation of NAM may be an "already established" communication platform in these cells, which is exacerbated by cholesterol dysregulation and likely by the therapeutic protocols.

NAM will, in turn, increase the exposure of the nucleus to hydrogen peroxide $\left(\mathrm{H}_{2} \mathrm{O}_{2}\right)$, superoxide $\left(\mathrm{O}_{2}^{-}\right)$, as well as hydroxyl radical $\left(\mathrm{OH}^{-}\right)$generated in excess by defective mitochondria overexpressing TSPO. The nucleus is usually out of reach of mitochondrial $\mathrm{O}_{2}{ }^{-}$ and $\mathrm{OH}^{-}$, which have very low estimated diffusion coefficients (55). The formation of NAM would bring mitochondria in a closer range to the nuclear DNA, facilitating the exposure of reactive species of the oxygen to the nuclear DNA (56), which maximize NF- $\mathrm{\kappa B}$ activation.

NAM may therefore represent both a defensive mechanism in response to apoptotic cues in the short term, as well as a detrimental coupling that compromises DNA integrity in the long one, leading to genomic alterations. This hypothesis ties well into the observations that cytoskeletal reorganization (which we have not here investigated) and cellular morphology are key factors in cellular resistance to apoptosis as well as in regulating the NF- $\mathrm{KB}$ signaling $(57,58)$. Equally, nuclear pleomorphism of tumors, proposed as a biomarker of malignancy for breast cancer (59), and the associated somatic mutations 
could be the consequence of the continuous exploitation of this localized, but nuclear-focused stress signaling.

Mitochondrial repositioning is nonetheless secondary to inefficient mitochondrial quality control for impaired mitophagy (60), which causes the accumulation of malfunctioning organelles and their bypassed respiratory products. TSPO suppresses PINK1-PARKINdriven mitophagy by preventing the ubiquitination of mitochondrial proteins (16). TSPO-rich mitochondria are therefore prone to repositioning on the nucleus, as they can systematically evade control by autophagy. The previously described deubiquitinating properties of TSPO (16) lead us to speculate that trafficking of TSPO onto the nuclear envelope could replicate a similar outcome in the nucleus by preventing nucleophagy (61) causing the undulated nuclear envelope observed ultrastructurally (fig. S2A). The alteration in lipid composition of the membranes to which TSPO localizes (mitochondria) or relocalizes (nucleus) could also be instrumental for this as well as to the altered organellar life cycle and protein interactome (Fig. 5H). Mitochondrial network remodeling, cholesterol sequestration on the perinuclear region, and cell death evasion are avoidable by the down-regulation of TSPO, its pharmacological inhibition, as well as the pharmacological activation of mitophagy. Furthermore, TSPO is overexpressed in mammary gland cell lines that have developed resistance to ET, thus conferring resistance to those who might instead be susceptible to the treatment (Fig. 4, D to G). Overexpression of the $\triangle$ TSPO-CRAC mutant, which fails binding of cholesterol by TSPO, is unable to replicate the same effect (Fig. 5G). These experiments propose cholesterol as an oncometabolite in the resistance primed by MRR, which is executed by physical reorganization of the mitochondrial network on the nucleus here reported.

\section{METHODS}

\section{Immunohistochemistry/immunocytochemistry}

Immunofluorescence: Cells were fixed in $4 \%$ paraformaldehyde (PFA) [10 min, room temperature (RT)] followed by three 5-min washes in phosphate-buffered saline (PBS). Permeabilization was performed with $0.5 \%$ Triton-X in PBS (10 min at RT) followed by washing. Blocking was carried out for 1 hour at RT in $10 \%$ goat serum and $3 \%$ bovine serum albumin in PBS. Primary antibody incubations were conducted overnight for 16 hours at $4^{\circ} \mathrm{C}$ in blocking solution as described. After a further wash step, secondary antibodies were incubated for 1 hour in blocking solution, before a final wash step. Cells were then mounted on slides with $4^{\prime}, 6$-diamidino-2phenylindole (DAPI) mounting medium (Abcam, ab104139). Cells were stained with the following primary antibodies: ATPB (1:400; Abcam, ab14730); lamin B2 (1:500; Abcam, ab8983); TSPO (1:200; Abcam, ab109497), NF-кB (1:500; Abcam, ab16502); ATAD3 (1:1000; gift from I. Holt, Biodonista), LXR $\beta$ (1:400; Abcam, ab28479), and STAR (1:500; Abcam, ab58013), and the following secondary antibodies: $\alpha$-mouse Alexa 555 (1:1000; Life Technologies, A21424) and $\alpha$-rabbit Alexa 488 (1:1000; Life Technologies, A11008).

Single dye immunofluorescence was used to stain paraffin sections of human mammary tissues with different TSPO antibody (ab109497)/NF- $\mathrm{\kappa B}$ (ab32360). The binding of mouse primary antibodies was detected using Alexa Fluor 488 (or 594) fluorochromeconjugated goat anti-mouse IgG (immunoglobulin G). Tissue sections were mounted with Fluoroshield Mounting Medium with DAPI to show.

\section{Cell culture}

MDA-MB-231 and MCF-7 human breast cancer cell lines, CF41 canine mammary line, K248P feline mammary line, WT, and Mitofusin $2^{-/-}$cell lines were all maintained at $37^{\circ} \mathrm{C}$ under humidified conditions and $5 \% \mathrm{CO}_{2}$ and grown in Dulbecco's modified Eagle's medium (Life Technologies, 41966-052) supplemented with 10\% fetal bovine serum (Life Technologies, 10082-147), penicillin (100 U/ml), and streptomycin (100 mg/ml) (Life Technologies, 15140-122). K248P cells were additionally supplemented with insulin $(10 \mu \mathrm{g} / \mathrm{ml})$ (Sigma-Aldrich). Cells were transiently transfected with genes of interest or small interfering RNA as described previously $(13,15)$ or using the manufacturer's instructions for Lipofectamine 3000 (Thermo Fisher Scientific, 18324010). Mnf2KO MEFs were generously provided by Prof. L. Scorrano (University of Padua, Italy).

\section{Cell fractionation}

Cells were lysed in cold isotonic buffer [ $250 \mathrm{mM}$ sucrose, $10 \mathrm{mM} \mathrm{KCl}$, $1.5 \mathrm{mM} \mathrm{MgCl}_{2}, 1$ mM EDTA, 1 mM EGTA, 20 mM Hepes, (pH 7.4)] containing protease inhibitor cocktail (Roche, 05892791001) by passing through a 26 -gauge needle 10 times using a 1 -ml syringe, followed by 20 -min incubation on ice. Nuclei were separated by centrifugation at $800 \mathrm{~g}$ for $5 \mathrm{~min}$ at $4^{\circ} \mathrm{C}$. For nuclear fractions, pellets were washed once in isotonic buffer followed by centrifugation $800 \mathrm{~g}$ for $10 \mathrm{~min}$ at $4^{\circ} \mathrm{C}$. Pellets were suspended in standard lysis buffer $[150 \mathrm{mM} \mathrm{NaCl}$, $1 \% \mathrm{v} / \mathrm{v}$ Triton X-100, $20 \mathrm{mM}$ tris (pH 7.4)] plus 10\% glycerol and $0.1 \%$ SDS and sonicated for $5 \mathrm{~s}$ to dissolve pellet. For mitochondrial fractions, supernatants were transferred to fresh tubes and centrifuged at $10,000 \mathrm{~g}$ for $10 \mathrm{~min}$ at $4^{\circ} \mathrm{C}$. Subsequent supernatants were collected as the cytosolic fractions, while mitochondrial pellets were washed once in cold isotonic buffer and then centrifuged at $10,000 \mathrm{~g}$ for $10 \mathrm{~min}$ at $4^{\circ} \mathrm{C}$. Last, mitochondrial pellets were lysed in lysis for $30 \mathrm{~min}$ on ice.

\section{Western blot}

Sample proteins were quantified using a BCA Protein Assay Kit (Thermo Fisher Scientific, 13276818). Equal amounts of protein (10 to $30 \mu \mathrm{g}$ for whole-cell lysates/cytosolic fractions; $10 \mu \mathrm{g}$ for mitochondrial nuclear fractions) were resolved on $12 \%$ SDS-polyacrylamide gel electrophoresis and transferred to nitrocellulose membranes (Thermo Fisher Scientific, 10339574). The membranes were blocked in $3 \%$ nonfat dry milk in TBST [ $50 \mathrm{mM}$ tris, $150 \mathrm{mM} \mathrm{NaCl}, 0.05 \%$ Tween 20, (pH 7.5)] for 1 hour and then incubated with the appropriate diluted primary antibody at $4^{\circ} \mathrm{C}$ overnight: LC3 (1:1000; Abcam, ab48394), TSPO (1:5000; Abcam, ab109497), actin (1:2000; ab8266), ATPB (1:5000; Abcam, ab14730), BAX (1:1000; Abcam, ab32503), Cyt c (1:1000; ab13575),

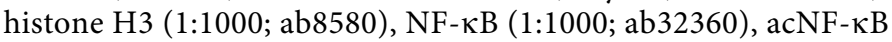
(1:500; ab19870), SIRT-1 (1:1000; ab110304), lamin B1 (1:1000; ab16048), and MTCO1 (1:1000; ab14705). Membranes were washed in TBST $(3 \times 15 \mathrm{~min}$ at RT) and then incubated with corresponding peroxidase-conjugated secondary antibodies (Dako, P0447, P0448) for 1 hour at RT. After further washing in TBST, blots were developed using an ECL Plus on Western Blotting Detection Kit (Thermo Fisher Scientific, 12316992). Immunoreactive bands were analyzed by performing densitometry with ImageJ software.

\section{Confocal imaging/ImageJ}

Fluorescence labeling was observed through an LSM 5 Pascal confocal microscope (Zeiss, Oberkochen, Germany), and images were recorded with the Pascal software (Zeiss). All image analyses were done using Fiji (ImageJ; NCBI, USA) and corresponding plug-ins. 
All staining was checked for nonspecific antibody labeling using control samples without primary antibody. None of the controls showed any signs of nonspecific fluorescence. For immunocytochemistry (ICC) and immunohistochemistry (IHC) imaging, single optical sections were analyzed. The infiltrate analysis was done using orthogonal rendering $3 \mathrm{D}$ optical stacks and counting the number of infiltrates per cell nucleus. The mitochondrial network analysis was performed using the "skeletonize" plug-in. Higher-resolution Airyscan processed images were acquired using an LSM 880 confocal with Airyscan (Carl Zeiss, Jena, Germany) system with GaAsP detectors and a module for Airyscan imaging. In Airyscan modes, a 63× Plan Apochromat (1.4 NA) oil objective was used. Confocal imaging was sequential for different fluorophore channels to obtain a series of axial images. Images of cells were optimized for visualization by manually adjusting the minimum and maximum intensity values of the histogram in the Zen software (Carl Zeiss). Images were analyzed using the variance filter and quantified by colocalization of fluorophores.

\section{Cell proliferation assays}

Cell proliferation assays were performed using the WST-1 Cell Proliferation Assay Kit (ab65473) and assessed on a standard plate reader (Tecan Sunrise).

\section{Cell death assays}

Cells were fixed in 4\% PFA for $10 \mathrm{~min}$ at RT followed by three 5 -min washes in PBS. Permeabilization was performed with $0.2 \%$ Triton X-100 in PBS for $10 \mathrm{~min}$ at RT followed by washing. To assess apoptosisassociated DNA fragmentation, a TUNEL Assay Kit (ab66108) was used according to instructions by the manufacturer. Alternatively, cells were live stained with Hoechst 33342 (Sigma-Aldrich, H6024) and propidium iodide (Sigma-Aldrich, 25535) to mark apoptotic cells and imaged on an inverted fluorescence microscope.

\section{Fluorescence imaging}

Cells were incubated with $5 \mu \mathrm{M}$ dihydroethidium (DHE; Life Technologies, D-1168) or $5 \mu \mathrm{M}$ MitoSOX (Life Technologies, M-36008) in recording media $\left[125 \mathrm{mM} \mathrm{NaCl}, 5 \mathrm{mM} \mathrm{KCl}, 1 \mathrm{mM} \mathrm{NaH}_{2} \mathrm{PO}_{4}, 20 \mathrm{mM}\right.$ Hepes, $5.5 \mathrm{mM}_{\text {glucose, }} 5 \mathrm{mM} \mathrm{NaHCO}_{3}$, and $1 \mathrm{mM} \mathrm{CaCl}_{2}$, (pH 7.4)] for $30 \mathrm{~min}$ at $37^{\circ} \mathrm{C}$. Cells were washed once in recording medium and then transferred to a Leica SP- 5 confocal microscope $(63 \times$ oil objective lens) for imaging, and fluorescence intensity was measured through continuous recording for at least $10 \mathrm{~min}$. Settings were kept constant between experiments. Mitochondrial regions of interest were selected, and the corresponding fluorescence intensities were calculated. For assay of cholesterol transport, Ergosta (dehydroergosterol-ergosta$5,7,9(11), 22$-tetraen-3 $\beta$-ol) was prepared and loaded as previously described (62). Briefly, Ergosta was added to an aqueous solution of $\mathrm{M} \beta C D$ ( $3 \mathrm{mM}$ Ergosta and $30 \mathrm{mM} \mathrm{M} \beta C D$ ). This mixture was overlaid with nitrogen, continuously vortexed under light protection for 24 hours at RT, and filtered through a $0.2-\mu \mathrm{m}$ filter to remove insoluble material and Ergosta crystals. Then, $20 \mu \mathrm{g}$ of DHE was added to the cells in the form of DHE-M $\beta C D$ complexes and allowed to incubate for $90 \mathrm{~min}$ at RT in PBS. Before imaging, cells were washed three times with culture media before being incubated with $20 \mathrm{nM}$ MitoTracker Red CMXRos. Cells were washed and then imaged.

\section{Statistical analysis}

Data are presented as means \pm SD of the mean. One-way analysis of variance (ANOVA) was used in multiple-group comparisons with
Bonferroni's post hoc test to compare two datasets within the group, and $P<0.05$ was considered significant. All analyses were performed in Microsoft Office Excel 2010 and GraphPad Prism 7. ${ }^{*} P \leq$ $0.05,{ }^{* *} P \leq 0.01,{ }^{* * *} P \leq 0.001$.

\section{Preparation of cells for electron microscopy (TEM and Immunogold TEM)}

For TEM analysis, MDA-MB-231 cells (control and treated with $0.5 \mu \mathrm{M}$ for 16 hours) were fixed at RT with $2.5 \%$ glutaraldehyde (v/v) in $0.1 \mathrm{M}$ sodium cacodylate buffer ( $\mathrm{pH} 7.4$ ) for 3 hours. The cells were pelleted by centrifugation, washed in buffer, and postfixed for 1 hour with $1 \%$ osmium tetroxide in $0.1 \mathrm{M}$ sodium cacodylate buffer. Samples were thoroughly rinsed, dehydrated in a graded series of ethanol, and embedded in epoxy resin (TAAB 812). Ultrathin sections (70 to $90 \mathrm{~nm}$ ) were cut using a Leica UC7 ultramicrotome mounted on 150 mesh copper grids and contrasted using UranyLess (TAAB) and 3\% Reynolds lead citrate (TAAB). Sections were examined at $120 \mathrm{kV}$ on a JEOL JEM-1400Plus TEM fitted with a RUBY digital camera (2kx2k). For TEM immunogold labeling, cell samples were fixed with $4 \%(\mathrm{w} / \mathrm{v})$ PFA, $0.1 \%$ (v/v) glutaraldehyde in $0.1 \mathrm{M}$ phosphate buffer ( $\mathrm{pH}$ 7.4) for 4 hours at RT, and spun down on $20 \%$ gelatine. Gelatine cell pellets were cryoprotected by incubating in $2.3 \mathrm{M}$ sucrose overnight at $4^{\circ} \mathrm{C}$. Gelatine blocks containing cells were cut further into 1- to 2-mm cubes, mounted on aluminum pins, and cryofixed by plunging into liquid nitrogen. Samples were stored in liquid nitrogen before cryosectioning. Ultrathin sections (70- to 90-nm thick) were cut using a Leica EM FC6 cryo-ultramicrotome and mounted on Pioloform film-supported nickel grids according to the Tokuyasu method. Sections were immunolabeled using anti-TSPO (1:200) (Abcam, ab109497) followed by a 12-nm colloidal gold anti-rabbit secondary antibody (1:40) (Jackson ImmunoResearch). Grids were examined at $120 \mathrm{kV}$ on a JEOL JEM-1400Plus TEM fitted with a RUBY digital camera ( $2 \mathrm{k} \times 2 \mathrm{k})$.

\section{SUPPLEMENTARY MATERIALS}

Supplementary material for this article is available at http://advances.sciencemag.org/cgi/ content/full/6/51/eabc9955/DC1

View/request a protocol for this paper from Bio-protocol.

\section{REFERENCES AND NOTES}

1. P. M. Quirós, A. Mottis, J. Auwerx, Mitonuclear communication in homeostasis and stress. Nat. Rev. Mol. Cell Biol. 17, 213-226 (2016).

2. R. A. Butow, N. G. Avadhani, Mitochondrial signaling: The retrograde response. Mol. Cell 14, 1-15 (2004).

3. U. Topf, L. Wrobel, A. Chacinska, Chatty mitochondria: Keeping balance in cellular protein homeostasis. Trends Cell Biol. 26, 577-586 (2016).

4. M. Guha, S. Srinivasan, G. Ruthel, A. K. Kashina, R. P. Carstens, A. Mendoza, C. Khanna, T. Van Winkle, N. G. Avadhani, Mitochondrial retrograde signaling induces epithelialmesenchymal transition and generates breast cancer stem cells. Oncogene 33, 5238-5250 (2014).

5. T. Carden, B. Singh, V. Mooga, P. Bajpai, K. K. Singh, Epigenetic modification of miR-663 controls mitochondria-to-nucleus retrograde signaling and tumor progression. J. Biol. Chem. 292, 20694-20706 (2017).

6. S. Feske, H. Okamura, P. G. Hogan, A. Rao, $\mathrm{Ca}^{2+} /$ calcineurin signalling in cells of the immune system. Biochem. Biophys. Res. Commun. 311, 1117-1132 (2003).

7. T. Arnould, S. Vankoningsloo, P. Renard, A. Houbion, N. Ninane, C. Demazy, J. Remacle, $M$. Raes, CREB activation induced by mitochondrial dysfunction is a new signaling pathway that impairs cell proliferation. EMBO J. 21, 53-63 (2002).

8. R. M. Monaghan, R. G. Barnes, K. Fisher, T. Andreou, N. Rooney, G. B. Poulin, A. J. Whitmarsh, A nuclear role for the respiratory enzyme CLK-1 in regulating mitochondrial stress responses and longevity. Nat. Cell Biol. 17, 782-792 (2015).

9. M. D. Cardamone, B. Tanasa, C. T. Cederquist, J. Huang, K. Mahdaviani, W. Li, M. G. Rosenfeld, M. Liesa, V. Perissi, Mitochondrial retrograde signaling in mammals is 
mediated by the transcriptional cofactor GPS2 via direct mitochondria-to-nucleus translocation. Mol. Cell 69, 757-772.e7 (2018).

10. Z. Liu, T. Sekito, M. Spírek, J. Thornton, R. A. Butow, Retrograde signaling is regulated by the dynamic interaction between Rtg2p and Mks1p. Mol. Cell 12, 401-411 (2003).

11. L. Scorrano, M. A. De Matteis, S. Emr, F. Giordano, G. Hajnóczky, B. Kornmann, L. L. Lackner, T. P. Levine, L. Pellegrini, K. Reinisch, R. Rizzuto, T. Simmen, H. Stenmark, C. Ungermann, M. Schuldiner, Coming together to define membrane contact sites. Nat. Commun. 20, 1287 (2019).

12. D. C. Wallace, Mitochondria and cancer. Nat. Rev. Cancer 12, 685-698 (2012).

13. D. Faccenda, C. H. Tan, A. Seraphim, M. R. Duchen, M. Campanella, IF1 limits the apoptotic-signalling cascade by preventing mitochondrial remodelling. Cell Death Differ. 20, 686-697 (2013).

14. L. Formentini, M. Sánchez-Aragó, L. Sánchez-Cenizo, J. M. Cuezva, The mitochondrial ATPase inhibitory factor 1 triggers a ROS-mediated retrograde prosurvival and proliferative response. Mol. Cell 6, 731-742 (2012).

15. D. Faccenda, J. Nakamura, G. Gorini, G. K. Dhoot, M. Piacentini, M. Yoshida, M. Campanella, Control of mitochondrial remodeling by the ATPase inhibitory factor 1 unveils a pro-survival relay via OPA1. Cell Rep. 18, 1869-1883 (2017).

16. J. Gatliff, M. Campanella, TSPO: Kaleidoscopic 18-kDa amid biochemical pharmacology, control and targeting of mitochondria. Biochem. J. 15, 107-121 (2016).

17. H. Miettinen, J. Kononen, H. Haapasalo, P. Helen, P. Sallinen, T. Harjuntausta, H. Helin, H. Alho, Expression of peripheral-type benzodiazepine receptor and diazepam binding inhibitor in human astrocytomas: Relationship to cell proliferation. Cancer Res. 55, 2691-2695 (1995).

18. K. Maaser, P. Grabowski, Y. Oezdem, A. Krahn, B. Heine, H. Stein, H. Buhr, M. Zeitz, $\mathrm{H}$. Scherübl, Up-regulation of the peripheral benzodiazepine receptor during human colorectal carcinogenesis and tumor spread. Clin. Cancer Res. 11, 1751-1756 (2005).

19. J. Zheng, R. Boisgard, K. Siquier-Pernet, D. Decaudin, F. Dollé, B. Tavitian, Differential expression of the $18 \mathrm{kDa}$ translocator protein (TSPO) by neoplastic and inflammatory cells in mouse tumors of breast cancer. Mol. Pharm. 8, 823-832 (2011).

20. D. Tang, J. Li, M. L. Nickels, G. Huang, A. S. Cohen, H. C. Manning, Preclinical evaluation of a novel TSPO PET Ligand 2-(7-Butyl-2-(4-(2-[ ${ }^{18}$ F]Fluoroethoxy)phenyl)-5Methylpyrazolo[1,5-a]Pyrimidin-3 -yl)- $N, N$-Diethylacetamide $\left({ }^{18} \mathrm{~F}\right.$-VUIIS1018A) to image glioma. Mol. Imaging Biol. 21, 113-121 (2018).

21. M. N. Tantawy, H. C. Manning, T. E. Peterson, D. C. Colvin, J. C. Gore, W. Lu, Z. Chen, C. C. Quarles, Translocator protein PET imaging in a preclinical prostate cancer model. Mol. Imaging Biol. 20, 200-204 (2018).

22. J. Gatliff, D. East, J. Crosby, R. Abeti, R. Harvey, W. Craigen, P. Parker, M. Campanella, TSPO interacts with VDAC1 and triggers a ROS-mediated inhibition of mitochondrial quality control. Autophagy 10, 2279-2296 (2014).

23. J. Gatliff, D. A. East, A. Singh, M. S. Alvarez, M. Frison, I. Matic, C. Ferraina, N. Sampson, F. Turkheimer, M. Campanella, A role for TSPO in mitochondrial $\mathrm{Ca}^{2+}$ homeostasis and redox stress signaling. Cell Death Dis. 8, e2896 (2017).

24. J. Fan, J. Liu, M. Culty, V. Papadopoulos, Acyl-coenzyme A binding domain containing 3 (ACBD3; PAP7; GCP60): An emerging signaling molecule. Prog. Lipid Res. 3, 218-234 (2010).

25. J. Lu, W. Wang, Y. Mi, C. Zhang, H. Ying, L. Wang, Y. Wang, L. Myatt, K. Sun, AKAP95mediated nuclear anchoring of PKA mediates cortisol-induced PTGS2 expression in human amnion fibroblasts. Sci. Signal. 10, eaac6160 (2017).

26. C. Yapo, A. G. Nair, J. H. Kotaleski, P. Vincent, L. R. V. Castro, Switch-like PKA responses in the nucleus of striatal neurons. J. Cell Sci. 131, jcs216556 (2018).

27. M. Hardwick, D. Fertikh, M. Culty, H. Li, B. Vidic, V. Papadopoulos, Peripheral-type benzodiazepine receptor (PBR) in human breast cancer: Correlation of breast cancer cell aggressive phenotype with PBR expression, nuclear localization, and PBR-mediated cell proliferation and nuclear transport of cholesterol. Cancer Res. 59, 831-842 (1999).

28. A. Beinlich, R. Strohmeier, M. Kaufmann, H. Kuhl, Relation of cell proliferation to expression of peripheral benzodiazepine receptors in human breast cancer cell lines. Biochem. Pharmacol. 60, 397-402 (2000).

29. V. Papadopoulos, Y. Aghazadeh, J. Fan, E. Campioli, B. Zirkin, A. Midzak, Translocator protein-mediated pharmacology of cholesterol transport and steroidogenesis. Mol. Cell. Endocrinol. 408, 90-98 (2015).

30. M. B. Rone, J. Fan, V. Papadopoulos, Cholesterol transport in steroid biosynthesis: Role of protein-protein interactions and implications in disease states. Biochim. Biophys. Acta 1791, 646-658 (2009).

31. M. B. Rone, A. S. Midzak, L. Issop, G. Rammouz, S. Jagannathan, J. Fan, X. Ye, J. Blonder, T. Veenstra, V. Papadopoulos, Identification of a dynamic mitochondrial protein complex driving cholesterol import, trafficking, and metabolism to steroid hormones. Mol. Endocrinol. 26, 1868-1882 (2012).
32. J. Fan, V. Papadopoulos, Evolutionary origin of the mitochondrial cholesterol transport machinery reveals a universal mechanism of steroid hormone biosynthesis in animals. PLOS ONE 8, e76701 (2013).

33. V. T. Nguyen, I. Barozzi, M. Faronato, Y. Lombardo, J. H. Steel, N. Patel, P. Darbre, L. Castellano, B. Győrffy, L. Woodley, A. Meira, D. K. Patten, V. Vircillo, M. Periyasamy, S. Ali, G. Frige, S. Minucci, R. C. Coombes, L. Magnani, Differential epigenetic reprogramming in response to specific endocrine therapies promotes cholesterol biosynthesis and cellular invasion. Nat. Commun. 6, 10044 (2015).

34. L. Magnani, G. Frige, R. M. Gadaleta, G. Corleone, S. Fabris, M. H. Kempe, P. J. Verschure, I. Barozzi, V. Vircillo, S.-P. Hong, Y. Perone, M. Saini, A. Trumpp, G. Viale, A. Neri, S. Ali, M. A. Colleoni, G. Pruneri, S. Minucci, Acquired CYP19A1 amplification is an early specific mechanism of aromatase inhibitor resistance in ER $\alpha$ metastatic breast cancer. Nat. Genet. 49, 444-450 (2017).

35. S. Khan, Z. Lopez-Dee, R. Kumar, J. Ling, Activation of NFkB is a novel mechanism of pro-survival activity of glucocorticoids in breast cancer cells. Cancer Lett. 337, 90-95 (2013).

36. J. Ling, R. Kumar, Crosstalk between NFkB and glucocorticoid signaling: A potential target of breast cancer therapy. Cancer Lett. 322, 119-126 (2012).

37. D. A. East, F. Fagiani, J. Crosby, N. D. Georgakopoulos, H. Bertrand, M. Schaap, A. Fowkes, G. Wells, M. Campanella, PMI: $A \Delta \Psi \mathrm{m}$ independent pharmacological regulator of mitophagy. Chem. Biol. 21, 1585-1596 (2014).

38. N. D. Georgakopoulos, M. Frison, M. S. Alvarez, H. Bertrand, G. Wells, M. Campanella, Reversible Keap1 inhibitors are preferential pharmacological tools to modulate cellular mitophagy. Sci. Rep. 7, 10303 (2017).

39. L. Jaremko, M. Jaremko, K. Giller, S. Becker, M. Zweckstetter, Structure of the mitochondrial translocator protein in complex with a diagnostic ligand. Science 343, 1363-1366 (2014).

40. T. Tenev, K. Bianchi, M. Darding, M. Broemer, C. Langlais, F. Wallberg, A. Zachariou, J. Lopez, M. M. Farlane, K. Cain, P. Meier, The Ripoptosome, a signaling platform that assembles in response to genotoxic stress and loss of IAPs. Mol. Cell 43, 432-448 (2011).

41. M. H. Jeng, M. A. Shupnik, T. P. Bender, E. H. Westin, D. Bandyopadhyay, R. Kumar, S. Masamura, R. J. Santen, Estrogen receptor expression and function in long-term estrogen-deprived human breast cancer cells. Endocrinology 139, 4164-4174 (1998).

42. R. J. Santen, E. K. Lobenhofer, C. A. Afshari, Y. Bao, R. X. Song, Adaptation of estrogenregulated genes in long-term estradiol deprived MCF-7 breast cancer cells. Breast Cancer Res. Treat. 94, 213-223 (2005).

43. T. J. Haley, A review of the literature of rotenone, 1,2,12,12a-tetrahydro-8,9-dimethoxy-2(1-methylethenyl)-1-benzopyrano[3,5-b]furo[2,3-h][1]benzopyran-6(6h)-one. J. Environ. Pathol. Toxicol. 1, 315-337 (1978).

44. O. de Brito, L. Scorrano, Mitofusin 2 tethers endoplasmic reticulum to mitochondria. Nature 456, 605-610 (2008).

45. G. Marwarha, S. Raza, K. Hammer, O. Ghribi, 27-hydroxycholesterol: A novel player in molecular carcinogenesis of breast and prostate cancer. Chem. Phys. Lipids 207, 108-126 (2017).

46. A. Kloudova, F. P. Guengerich, P. Soucek, The role of oxysterols in human cancer. Trends Endocrinol. Metab. 28, 485-496 (2017).

47. F. Bovenga, C. Sabbà, A. Moschetta, Uncoupling nuclear receptor LXR and cholestero metabolism in cancer. Cell Metab. 21, 517-526 (2015).

48. M. Modzel, F. W. Lund, D. Wüstner, Synthesis and live-cell imaging of fluorescent sterols for analysis of intracellular cholesterol transport. Methods Mol. Biol. 1583, 111-140 (2017).

49. D. Strobbe, M. Campanella, Anxiolytic therapy: A paradigm of successful mitochondrial pharmacology. Trends Pharmacol. Sci. 39, 437-439 (2018).

50. H. Yang, W. Zhang, H. Pan, H. G. Feldser, E. Lainez, C. Miller, S. Leung, Z. Zhong, H. Zhao, S. Sweitzer, T. Considine, T. Riera, V. Suri, B. White, J. L. Ellis, G. P. Vlasuk, C. Loh, SIRT1 activators suppress inflammatory responses through promotion of $\mathrm{p} 65$ deacetylation and inhibition of NF-KB activity. PLOS ONE 7, e46364 (2012).

51. F. Yeung, J. E. Hoberg, C. S. Ramsey, M. D. Keller, D. R. Jones, R. A. Frye, M. W. Mayo, Modulation of NF-KB-dependent transcription and cell survival by SIRT1 deacetylase. EMBO J. 23, 2369-2380 (2004).

52. M. D. Tambini, M. Pera, E. Kanter, H. Yang, C. Guardia-Laguarta, D. Holtzman, D. Sulzer, E. Area-Gomez, E. A. Schon, ApoE4 upregulates the activity of mitochondria-associated ER membranes. EMBO Rep. 17, 27-36 (2016).

53. J. Nunnari, A. Suomalainen, Mitochondria: In sickness and in health. Cell 148, 1145-1159 (2012).

54. J. Fan, P. Lindemann, M. G. J. Feuilloley, V. Papadopoulos, Structural and functional evolution of the translocator protein (18 kDa). Curr. Mol. Med. 12, 369-386 (2012).

55. A. R. Cardoso, B. Chausse, F. M. da Cunha, L. A. Luévano-Martínez, T. B. M. Marazzi, P. S. Pessoa, B. B. Queliconi, A. J. Kowaltowski, Mitochondrial compartmentalization of redox processes. Free Radic. Biol. Med. 52, 2201-2208 (2012).

56. K. Ishikawa, K. Takenaga, M. Akimoto, N. Koshikawa, A. Yamaguchi, H. Imanishi, K. Nakada, Y. Honma, J.-I. Hayashi, ROS-generating mitochondrial DNA mutations can regulate tumor cell metastasis. Science 320, 661-664 (2008). 
57. W. Spencer, H. Kwon, P. Crépieux, N. Leclerc, R. Lin, J. Hiscott, Taxol selectively blocks microtubule dependent NF- $\kappa B$ activation by phorbol ester via inhibition of $1 \kappa B \alpha$ phosphorylation and degradation. Oncogene 18, 495-505 (1999).

58. S. Becker-Weimann, G. Xiong, S. Furuta, J. Han, I. Kuhn, U.-D. Akavia, D. Pe'er, M. J. Bissell $\mathrm{R}$. Xu, NFkB disrupts tissue polarity in 3D by preventing integration of microenvironmental signals. Oncotarget 4, 2010-2020 (2013).

59. G. Bussolati, C. Marchiò, L. Gaetano, R. Lupo, A. Sapino, Pleomorphism of the nuclear envelope in breast cancer: A new approach to an old problem. J. Cell. Mol. Med. 12 209-218 (2008).

60. G. Twig, O. S. Shirihai, The interplay between mitochondrial dynamics and mitophagy. Antioxid. Redox Signal. 14, 1939-1951 (2011).

61. Z. Dou, C. Xu, G. Donahue, T. Shimi, J.-A. Pan, J. Zhu, A. Ivanov, B. C. Capell, A. M. Drake, P. P. Shah, J. M. Catanzaro, M. D. Ricketts, T. Lamark, S. A. Adam, R. Marmorstein, W.-X. Zong, T. Johansen, R. D. Goldman, P. D. Adams, S. L. Berger, Autophagy mediates degradation of nuclear lamina. Nature 527, 105-109 (2015).

62. A. L. McIntosh, B. P. Atshaves, H. Huang, A. M. Gallegos, A. B. Kier, F. Schroeder, Fluorescence techniques using dehydroergosterol to study cholesterol trafficking. Lipids 43, 1185-1208 (2008)

\section{Acknowledgments}

Funding: The research activities lead by M.C. are supported by the following funders, who are gratefully acknowledged: Biotechnology and Biological Sciences Research Council (grant numbers BB/M010384/1 and BB/N007042/1), Rotary Foundation, the Petplan Charitable Trust (Project references: S12-14 and 373-411) and the European Research Council COG
2018-819600 FIRM. Author contributions: M.C. conceived, designed, and coordinated the project together with R.D. and D.A.E. M.C., R.D., D.A.E., L.K.H., D.F., J.C., M.S.A., and M.R. performed the experiments and ran the analysis. A.S. generated TSPO mutants. R.A.F. and G.V.-B. performed the EM analysis, while G.D. and M.M. performed the immunohistochemistry analysis, on which guidance was obtained by K.S. and V.Z.; R.B., G.S., and D.X. worked out the bioinformatics data. R.A. run and analyzed the biochemical experiments on Sirtuin-1. L.E.R. and J.P.C. generated and analyzed the data obtained with the Airyscan confocal. I.C. and A.H.-B. contributed the EM data in MCF-7 cells. All authors have critically reviewed the manuscript and advised accordingly. Conflict of interests: The authors declare that they have no competing interests. Data and materials availability: All data needed to evaluate the conclusions in the paper are present in the paper and/or the Supplementary Materials. Additional data related to this paper may be requested from the authors.

Submitted 27 May 2020

Accepted 13 November 2020

Published 18 December 2020

$10.1126 /$ sciadv.abc9955

Citation: R. Desai, D. A. East, L. Hardy, D. Faccenda, M. Rigon, J. Crosby, M. S. Alvarez, A. Singh, M. Mainenti, L. K. Hussey, R. Bentham, G. Szabadkai, V. Zappulli, G. Dhoot, L. E. Romano, D. Xia, I. Coppens, A. Hamacher-Brady, J. P. Chapple, R. Abeti, R. A. Fleck, G. Vizcay-Barrena, K. Smith, M. Campanella, Mitochondria form contact sites with the nucleus to couple prosurvival retrograde response. Sci. Adv. 6, eabc9955 (2020). 


\section{ScienceAdvances}

\section{Mitochondria form contact sites with the nucleus to couple prosurvival retrograde response}

Radha Desai, Daniel A. East, Liana Hardy, Danilo Faccenda, Manuel Rigon, James Crosby, María Soledad Alvarez, Aarti Singh, Marta Mainenti, Laura Kuhlman Hussey, Robert Bentham, Gyorgy Szabadkai, Valentina Zappulli, Gurtej K. Dhoot, Lisa E. Romano, Dong Xia, Isabelle Coppens, Anne Hamacher-Brady, J. Paul Chapple, Rosella Abeti, Roland A. Fleck, Gema Vizcay-Barrena, Kenneth Smith and Michelangelo Campanella

Sci Adv 6 (51), eabc9955.

DOI: $10.1126 /$ sciadv.abc9955

ARTICLE TOOLS

SUPPLEMENTARY

MATERIALS

REFERENCES

PERMISSIONS http://advances.sciencemag.org/content/6/51/eabc9955

http://advances.sciencemag.org/content/suppl/2020/12/14/6.51.eabc9955.DC1

This article cites 62 articles, 11 of which you can access for free http://advances.sciencemag.org/content/6/51/eabc9955\#BIBL

http://www.sciencemag.org/help/reprints-and-permissions

Science Advances (ISSN 2375-2548) is published by the American Association for the Advancement of Science, 1200 New York Avenue NW, Washington, DC 20005. The title Science Advances is a registered trademark of AAAS.

Copyright @ 2020 The Authors, some rights reserved; exclusive licensee American Association for the Advancement of Science. No claim to original U.S. Government Works. Distributed under a Creative Commons Attribution NonCommercial License 4.0 (CC BY-NC). 